OSU-HEP-08-08

FERMILAB-PUB-08-432-T

\title{
A Light Scalar as the Messenger of Electroweak and Flavor Symmetry Breaking
}

\author{
J. D. Lykken 1 , †, Z. Murdock 2,, , and S. Nand $3,+, \ddagger$, \\ $\dagger$ Theoretical Physics Department, Fermilab, P.O. Box 500, Batavia, IL 60510 \\ ${ }^{\ddagger}$ Department of Physics and Oklahoma Center for High Energy Physics, Oklahoma State University, \\ Stillwater, OK 74078
}

\begin{abstract}
We propose a new framework for understanding the hierarchies of fermion masses and mixings. The masses and mixings of all Standard Model (SM) charged fermions other than top arise from higher dimensional operators involving a messenger scalar $S$ and flavon scalars $F_{i}$. The flavons spontaneously break SM flavor symmetries at around the $\mathrm{TeV}$ scale. The SM singlet scalar $S$ couples directly to the Higgs $H$ and spontaneously breaks another $U(1)$ at the electroweak scale. At the TeV scale, SM quarks and charged leptons have renormalizable couplings to $S$, but not to $H$ or $F_{i}$. These couplings involve new heavy vectorlike fermions. Integrating out these fermions produces a pattern of higher dimensional operators that reproduce the observed hierarchies of the SM masses and mixings in terms of powers of the "little hierarchy": the ratio of the electroweak scale to the flavor-breaking scale.

The framework has important phenomenological implications. Flavor-changing neutral currents are within experimental limits but $D^{0}-\bar{D}^{0}$ mixing and $B_{s} \rightarrow \mu^{+} \mu^{-}$could be close to current sensitivities. The neutral scalar $s$ of the messenger field mixes with the light Higgs of the SM, which can have strong effects on Higgs decay branching fractions. The $s$ mass eigenstate may be lighter than the Higgs, and could be detected at the Tevatron or the LHC.
\end{abstract}

\section{Introduction}

Explaining the fermion mass hierarchy and mixing pattern is an outstanding challenge of particle physics [1] 2] 3. The fermion masses are parameterized by the Standard Model Yukawa interactions of chiral fermions with a single Higgs doublet. It is technically natural for the dimensionless Yukawa couplings to take small values, since global chiral flavor symmetries are restored (at tree level) in the limit that these couplings vanish, but it is a total mystery why these values are spread over more than five orders of magnitude, in a suggestive pattern of inter-generational and intra-generational hierarchies.

Although the gauge sector of the SM is well established, little is yet known about the Higgs sector. Higgs physics may be much richer than the minimal SM formulation, presenting new dynamics at the TeV scale

\footnotetext{
${ }^{1}$ Email address: lykken@fnal.gov

${ }^{2}$ zekemurdock@gmail.com

${ }^{3}$ Scientific visitor at Fermilab, e-mail address: s.nandi@okstate.edu
} 
that will be accessible to experiments at the LHC. Most work on extended Higgs sectors has been motivated by frameworks for understanding the naturalness and hierarchy problem of the SM Higgs boson, but not by the hierarchy problems of the SM flavor sector. One reason is that models that attempt to generate the flavor-breaking patterns of the SM Yukawas from new TeV scale dynamics are strongly constrained by experimental searches for flavor-changing neutral currents (FCNCs) and charged lepton flavor violation (CLFV).

The top quark Yukawa coupling has a value close to one, suggesting that a SM Yukawa coupling is the correct explanation for the top mass. The smallness of the other Yukawas suggests that some or all of the other quarks and the charged leptons do not couple directly to the electroweak symmetry breaking order parameter, which in the SM is represented by the vacuum expectation value (vev) of the Higgs scalar. Thus a good starting point to construct theories of flavor is to specify a field or mechanism to act as the messenger of electroweak symmetry breaking to the other quarks and leptons.

One simple choice for a messenger is a TeV mass scalar leptoquark, postulated to have a renomalizable coupling between the top quark and the SM leptons [4, 5. Radiative corrections can then generate a natural hierarchy of fermion masses related to powers of a loop factor.

An even simpler choice for a messenger is an electroweak mass scalar that transforms as a SM singlet and extends the Higgs sector of the SM. In this work, we explore this idea of an extended Higgs sector related to the generation of the fermion mass hierarchy. We present a simple framework where the Higgs doublet $H$ couples directly to a complex scalar $S$ that is a SM singlet and is charged under a new local $U(1)_{S}$ symmetry carried by a vector boson $Z^{\prime}$. All of the SM fermions are singlets under this new $U(1)_{S}$ (apart from small effects from $Z-Z^{\prime}$ mixing), which is broken spontaneously at the electroweak scale by the vacuum expectation value of $S$.

In our framework the singlet scalar $S$ is the messenger to SM fermions of both flavor breaking and electroweak symmetry breaking. All SM fermions apart from the third generation quark doublet $q_{3 L}$ and right-handed top $u_{3 R}$ are assumed to carry a nonzero charge under a gauged chiral flavor symmetry forbidding all SM dimension 4 Yukawa couplings except that of the top quark. We assume that the flavor symmetry is spontaneously broken at a scale $\gtrsim 1 \mathrm{TeV}$ by the vacuum expectation of one or more complex scalar "flavon" fields $F_{i}$. The flavor charges of the SM fermions forbid any dimension 4 couplings to either $F_{i}$ or to the Higgs field $H$.

We introduce new fermions that are vectorlike under both the SM gauge symmetries and $U(1)_{S}$; these fermions naturally acquire masses $\gtrsim \mathrm{TeV}$ that we will generically denote as $M$, and have dimension 4 couplings to both $F_{i}$ and to $H$. Integrating out these heavy fermions gives higher dimension effective couplings of the SM fermions to $H$ that replace the role of Yukawa couplings in the SM. These couplings contain explicit flavor breaking in the form of $\left\langle F_{i}\right\rangle / M$, which we take to be of order 1 , as well as being suppressed by powers of $S^{\dagger} S / M^{2}$, whose vev we take to be of order $1 / 50$.

In our framework all of the observed SM fermion mass hierarchies are generated from powers of $\langle S\rangle / M \sim$ $1 / 7$, which is essentially the ratio of the electroweak scale to the TeV scale, often called the "little hierarchy". We can be agnostic about the source of the little hierarchy itself, since many possibilities have been proposed. The additional challenge of our framework is to achieve simultaneously the appropriate flavon physics at the $\mathrm{TeV}$ scale.

Models in our framework have, in addition to the SM particle content, a light singlet scalar $s$ that mixes with the Higgs boson $h$. Exchanges of $s$ between SM fermions are a new source of FCNC. There is an extra $Z^{\prime}$ at the electroweak (EW) scale, but apart from small $Z-Z^{\prime}$ mixing effects it does not couple to SM fermions. There may be other $Z^{\prime}$ s and one or more flavon scalars at the TeV scale. We predict a host of new heavy fermions around the TeV scale; these are also a source of new FCNC and CLFV effects. We show that flavon charge patterns that reproduce the observed SM fermion masses and mixings also supply enough 
extra suppression of FCNC and CLFV effects to satisfy current experimental bounds.

In addition to explaining the hierarchy of fermion masses and mixings, models in our framework have many interesting phenomenological implications. Mixing of the singlet $s$ with the Higgs boson $h$ can cause large deviations from the SM predictions for the Higgs decay branching fractions, potentially observable at the Tevatron or LHC. The $s$ mass eigenstate itself will also be produced at the LHC, and could be confused with $h$ if it turns out to be the lightest mass eigenstate. While new FCNC effects are suppressed, we predict contributions to $D^{0}-\bar{D}^{0}$ mixing, $B_{s} \rightarrow \mu^{+} \mu^{-}$, that are close to the current value or limit. The exotic top quark decays $t \rightarrow c h$ and $t \rightarrow c s$ can have branching fractions on the order of $10^{-3}$.

Our paper is organized as follows. In section 2, we present the basic outline of our framework. In section 3 , we discuss the constraints on the model parameters from the low energy phenomenology. Section 4 contains the phenomenological implications and predictions of the model, especially for the new top decays and Higgs signals at the Tevatron and LHC. In section 5, we outline a possible ultraviolet completion realizing our proposal. Section 6 contains our conclusions and further discussion.

\section{Model and formalism}

We extend the gauge symmetry of the SM by a $U(1)_{S}$ local symmetry and an additional local flavon symmetry which in the simplest case would be a $U(1)_{F}$. All of the SM fermions are neutral with respect to $U(1)_{S}$, while all of the SM fermions apart from the third generation quark doublet $q_{3 L}$ and right-handed top $u_{3 R}$ are charged under the chiral $U(1)_{F}$. We introduce a complex scalar field $S$ which has charge 1 under $U(1)_{S}$, is neutral under the flavon symmetry, and is a SM singlet. We also introduce one or more complex scalar fields $F_{i}$, the "flavon" scalars. In the simplest case there would be a single flavon scalar $F$ that has charge 1 under $U(1)_{F}$, is neutral under $U(1)_{S}$, and is a SM singlet. The Higgs field $H$ is taken as neutral under $U(1)_{S} \times U(1)_{F}$. We assume that the flavon charges of the SM fermions are such that only the top quark has an allowed dimension 4 Yukawa interaction.

The $S$ field is assumed to develop a vev that spontaneously breaks the $U(1)_{S}$ symmetry. In frameworks where the little hierarchy between the electroweak scale and the $\mathrm{TeV}$ scale is generated, this could occur naturally by extending the Higgs sector to include $S$, with a mixed potential. The pseudoscalar component of $S$ is then "eaten" to give mass to the $U(1)_{S} Z^{\prime}$ gauge boson. Notice that the vev of $S$ does not in itself break any of the global flavor symmetries of the Yukawa-less SM; $S$ is only a messenger of flavor breaking, just as it is also a messenger of electroweak breaking. This is the fundamental distinction that allows $S$ to exist at the electroweak scale without inducing unacceptably large flavor violating effects.

The flavon scalars $F_{i}$ are assumed to develop vevs that spontaneously break the local flavon symmetry at the $\mathrm{TeV}$ scale, with the pseudoscalar components of the $F_{i}$ eaten to give the flavon gauge bosons mass. To preserve the little hierarchy, we assume that the direct mixing between the $F_{i}$ and the extended Higgs sector is negligible.

In this framework the Yukawa interactions of the lighter quarks and leptons are replaced by higher dimension operators that couple these fermions to $H, S$, and the $F_{i}$. As we will show later in an explicit example, these can be generated as effective couplings by integrating out new heavy fermions at the TeV scale. These effective couplings should respect all of the SM gauge symmetries, as well as $U(1)_{S}$ and the flavon symmetries. In particular, the $U(1)_{S}$ charged field $S$ can only appear as powers of $S^{\dagger} S / M^{2}$, where

$M$ denotes a generic TeV scale parameter. Powers of $F_{i} / M$ and $F_{i}^{\dagger} / M$ can also appear, but the exact form depends on the flavon charge assignments of the SM fermions. Since we will assume that vevs of the $F_{i}$ are of order $M$, we can absorb the $F_{i} / M$ dependence into the dimensionless complex couplings $h_{i j}$, where $i, j$ are generation labels; all these couplings we will then take to be of order 1. 
The observed SM fermion mass hierachy is generated from the following low energy effective interactions:

$$
\begin{aligned}
\mathcal{L}^{\text {Yuk }}= & h_{33}^{u} \bar{q}_{3 L} u_{3 R} \bar{H}+\left(\frac{S^{\dagger} S}{M^{2}}\right)\left(h_{33}^{d} \bar{q}_{3 L} d_{3 R} H+h_{22}^{u} \bar{q}_{2 L} u_{2 R} \bar{H}+h_{23}^{u} \bar{q}_{2 L} u_{3 R} \bar{H}+h_{32}^{u} \bar{q}_{3 L} u_{2 R} \bar{H}\right) \\
& +\left(\frac{S^{\dagger} S}{M^{2}}\right)^{2}\left(h_{22}^{d} \bar{q}_{2 L} d_{2 R} H+h_{23}^{d} \bar{q}_{2 L} d_{3 R} H+h_{32}^{d} \bar{q}_{3 L} d_{2 R} H+h_{12}^{u} \bar{q}_{1 L} u_{2 R} \bar{H}+h_{21}^{u} \bar{q}_{2 L} u_{1 R} \bar{H}\right. \\
& \left.+h_{13}^{u} \bar{q}_{1 L} u_{3 R} \bar{H}+h_{31}^{u} \bar{q}_{3 L} u_{1 R} \bar{H}\right)+\left(\frac{S^{\dagger} S}{M^{2}}\right)^{3}\left(h_{11}^{u} \bar{q}_{1 L} u_{1 R} \bar{H}+h_{11}^{d} \bar{q}_{1 L} d_{1 R} H\right. \\
& \left.+h_{12}^{d} \bar{q}_{1 L} d_{2 R} H+h_{21}^{d} \bar{q}_{2 L} d_{1 R} H+h_{13}^{d} \bar{q}_{1 L} d_{3 R} H+h_{31}^{d} \bar{q}_{3 L} d_{1 R} H\right)+ \text { h.c. }
\end{aligned}
$$

Note that the above interactions are very similar to those proposed in reference [6], except our interactions involve suppression by powers of $\left(\frac{S^{\dagger} S}{M^{2}}\right)$, instead of $\left(\frac{H^{\dagger} H}{M^{2}}\right)$. We will refer to this as the Babu-Nandi texture. The hierarchies among the fermion masses and mixings are obtained from a single small dimensionless parameter,

$$
\epsilon \equiv \frac{v_{s}}{M}
$$

where $v_{s}$ is the vev of $S$. As was shown in [6], a good fit to the observed fermion masses and mixings is obtained with $\epsilon \sim 0.15$. The couplings $h_{i j}$ are all of order one; the largest coupling needed is $h_{23}^{u}=1.4$, while the smallest coupling needed is $h_{22}^{u}=0.14$.

The Babu-Nandi texture is not unique, and it does not predict any precise fermion mass relations, since there are slightly more unspecified order 1 parameters than there are Yukawa parameters in the SM.

\subsection{Fermion masses and CKM mixing}

The gauge symmetry of our model is the usual $S U(3)_{c} \times S U(2)_{L} \times U(1)_{Y}$ of the SM, plus two additional local symmetries: $U(1)_{S}$ and the flavon symmetry. The SM symmetry is broken spontaneously by the usual Higgs doublet $H$ at the electroweak scale. We assume that the extra $U(1)_{S}$ symmetry is also broken spontaneously at the electroweak scale by a SM singlet complex scalar field $S$. The flavon symmetry, $U(1)_{F}$ in the simplest case, is broken spontaneously above a TeV by a SM singlet scalar flavon field $F$. The pseudoscalar part of the complex scalar field $S$ is absorbed by the $Z^{\prime}$ gauge boson $U(1)_{S}$ to get its mass. Thus after symmetry breaking the remaining scalars at the electroweak scale are neutral bosons $h$ and $s$. Parameterizing the Higgs doublet and singlet in the unitary gauge as

$$
H=\left(\begin{array}{c}
0 \\
\frac{h}{\sqrt{2}}+v
\end{array}\right) \quad S=\left(\frac{s}{\sqrt{2}}+v_{s}\right),
$$

with $v \simeq 174 \mathrm{GeV}$, and defining an additional small parameter

$$
\beta \equiv \frac{v}{M},
$$

we obtain, from eqs. (14 the following mass matrices for the up and down quark sector:

$$
M_{u}=\left(\begin{array}{ccc}
h_{11}^{u} \epsilon^{6} & h_{12}^{u} \epsilon^{4} & h_{13}^{u} \epsilon^{4} \\
h_{21}^{u} \epsilon^{4} & h_{22}^{u} \epsilon^{2} & h_{23}^{u} \epsilon^{2} \\
h_{31}^{u} \epsilon^{4} & h_{32}^{u} \epsilon^{2} & h_{33}^{u}
\end{array}\right) v, \quad M_{d}=\left(\begin{array}{ccc}
h_{11}^{d} \epsilon^{6} & h_{12}^{d} \epsilon^{6} & h_{13}^{d} \epsilon^{6} \\
h_{21}^{d} \epsilon^{6} & h_{22}^{d} \epsilon^{4} & h_{23}^{d} \epsilon^{4} \\
h_{31}^{d} \epsilon^{6} & h_{32}^{d} \epsilon^{4} & h_{33}^{d} \epsilon^{2}
\end{array}\right) v
$$


The charged lepton mass matrix is obtained from $M_{d}$ by replacing the couplings $h_{i j}$ appropriately. Note that these mass matrices are the same as in [6], and as was shown there, good fits to the quark and charged lepton masses, as well as the CKM mixing angles are obtained by choosing $\epsilon \sim 0.15$, and all the couplings $h_{i j}$ of order one. To leading order in $\epsilon$, the fermion masses are given by

$$
\begin{aligned}
& \left(m_{t}, m_{c}, m_{u}\right) \simeq\left(\left|h_{33}^{u}\right|,\left|h_{22}^{u}\right| \epsilon^{2},\left|h_{11}^{u}-h_{12}^{u} h_{21}^{u} / h_{22}^{u}\right| \epsilon^{6}\right) v, \\
& \left(m_{b}, m_{s}, m_{d}\right) \simeq\left(\left|h_{33}^{d}\right| \epsilon^{2},\left|h_{22}^{d}\right| \epsilon^{4},\left|h_{11}^{d}\right| \epsilon^{6}\right) v \\
& \left(m_{\tau}, m_{\mu}, m_{e}\right) \simeq\left(\left|h_{33}^{\ell}\right| \epsilon^{2},\left|h_{22}^{\ell}\right| \epsilon^{4},\left|h_{11}^{\ell}\right| \epsilon^{6}\right) v
\end{aligned}
$$

while the quark mixing angles are

$$
\begin{aligned}
\left|V_{u s}\right| & \simeq\left|\frac{h_{12}^{d}}{h_{22}^{d}}-\frac{h_{12}^{u}}{h_{22}^{u}}\right| \epsilon^{2}, \\
\left|V_{c b}\right| & \simeq\left|\frac{h_{23}^{d}}{h_{33}^{d}}-\frac{h_{23}^{u}}{h_{33}^{u}}\right| \epsilon^{2}, \\
\left|V_{u b}\right| & \simeq\left|\frac{h_{13}^{d}}{h_{33}^{d}}-\frac{h_{12}^{u} h_{23}^{d}}{h_{22}^{u} h_{33}^{d}}-\frac{h_{13}^{u}}{h_{33}^{u}}\right| \epsilon^{4} .
\end{aligned}
$$

Generically all of the $h_{i j}$ can be nonvanishing, but in a particular ultraviolet (UV) completion flavon charge conservation may push some of them to higher order in $\epsilon$ or to vanish altogether. However from (6) and (7) we see that the Babu-Nandi texture is rather robust: the only flavor off-diagonal couplings needed to reproduce the observed mixings are one or more of $h_{12}^{d}, h_{12}^{u}$, one or more of $h_{23}^{d}, h_{23}^{u}$, and one or more of $h_{13}^{d}, h_{13}^{u}$; the rest can either vanish or appear at higher order in $\epsilon$.

\subsection{Yukawa interactions and FCNC}

Our model has flavor changing neutral current interactions in the Yukawa sector. Using eqs.(1-4), the Yukawa interaction matrices $Y_{u}^{h}, Y_{d}^{h}, Y_{u}^{s}, Y_{d}^{s}$ for the up and down sector, for $h^{0}$ and $s^{0}$ fields are obtained to be

$$
\sqrt{2} Y_{u}^{h}=\left(\begin{array}{ccc}
h_{11}^{u} \epsilon^{6} & h_{12}^{u} \epsilon^{4} & h_{13}^{u} \epsilon^{4} \\
h_{21}^{u} \epsilon^{4} & h_{22}^{u} \epsilon^{2} & h_{23}^{u} \epsilon^{2} \\
h_{31}^{u} \epsilon^{4} & h_{32}^{u} \epsilon^{2} & h_{33}^{u}
\end{array}\right), \quad \sqrt{2} Y_{d}^{h}=\left(\begin{array}{ccc}
h_{11}^{d} \epsilon^{6} & h_{12}^{d} \epsilon^{6} & h_{13}^{d} \epsilon^{6} \\
h_{21}^{d} \epsilon^{6} & h_{22}^{d} \epsilon^{4} & h_{23}^{d} \epsilon^{4} \\
h_{31}^{d} \epsilon^{6} & h_{32}^{d} \epsilon^{4} & h_{33}^{d} \epsilon^{2}
\end{array}\right)
$$

with the charged lepton Yukawa coupling matrix $Y_{\ell}$ obtained from $Y_{d}$ by replaing $h_{i j}^{d} \rightarrow h_{i j}^{\ell}$.

$$
\sqrt{2} Y_{u}^{s}=\left(\begin{array}{ccc}
6 h_{11}^{u} \epsilon^{5} \beta & 4 h_{12}^{u} \epsilon^{3} \beta & 4 h_{13}^{u} \epsilon^{3} \beta \\
4 h_{21}^{u} \epsilon^{3} \beta & 2 h_{22}^{u} \epsilon \beta & 2 h_{23}^{u} \epsilon \beta \\
4 h_{31}^{u} \epsilon^{3} \beta & 2 h_{32}^{u} \epsilon \beta & 0
\end{array}\right), \quad \sqrt{2} Y_{d}^{s}=\left(\begin{array}{ccc}
6 h_{11}^{d} \epsilon^{5} \beta & 6 h_{12}^{d} \epsilon^{5} \beta & 6 h_{13}^{d} \epsilon^{5} \beta \\
6 h_{21}^{d} \epsilon^{5} \beta & 4 h_{22}^{d} \epsilon^{3} \beta & 4 h_{23}^{d} \epsilon^{3} \beta \\
6 h_{31}^{d} \epsilon^{5} \beta & 4 h_{32}^{d} \epsilon^{3} \beta & 2 h_{33}^{d} \epsilon \beta
\end{array}\right)
$$

with the charged lepton Yukawa coupling matrix $Y_{\ell}$ obtained from $Y_{d}$ by replaing $h_{i j}^{d} \rightarrow h_{i j}^{\ell}$.

There are several important features that distinguish our model from the proposals in [6, 7, 8]:

i) Note, from eqs.(5) and (8), in our model, the Yukawa couplings of h to the SM fermions are exactly the same as in the SM. This is because the fermion mass hierarchy in our model is arising from $\left(\frac{S^{\dagger} S}{M^{2}}\right)$. This 
is a distinguishing feature of our model from that proposed in [6, 7] where the Yukawa couplings of $h$ are flavor dependent, because the hierarchy there arises from $\left(\frac{H^{\dagger} H}{M^{2}}\right)$.

ii) In our model, we have an additional singlet Higgs boson whose couplings to the SM fermions are flavor dependent as given in eq. (9). Again, this is because the hierarchy in our model arises from $\left(\frac{S^{\dagger} S}{M^{2}}\right)$. In particular, $s^{0}$ does not couple to the top quark, and its dominant fermionic coupling is to the bottom quark. This will have interesting phenomenological implications for the Higgs searches at the LHC.

iii) We note from eqs. (518) that the mass matrices and the corresponding Yukawa coupling matrices for $h$ are proportional as in the SM. Thus there are no flavor changing Yukawa interactions mediated by $h$. However, this is not true for the Yukawa interactions of the singlet Higgs as can be seen from eqs. (5) and (9). Thus $s$ exchange will lead to flavor violation in the neutral Higgs interactions.

\subsection{Higgs sector and the $Z^{\prime}$}

The Higgs potential of our model, consistent with the SM and the extra $U(1)_{S}$ symmetry, can be written as

$$
V(H, S)=-\mu_{H}^{2}\left(H^{\dagger} H\right)-\mu_{S}^{2}\left(S^{\dagger} S\right)+\lambda_{H}\left(H^{\dagger} H\right)^{2}+\lambda_{S}\left(S^{\dagger} S\right)^{2}+\lambda_{H S}\left(H^{\dagger} H\right)\left(S^{\dagger} S\right) .
$$

Note that after absorbing the three components of $\mathrm{H}$ in $W^{ \pm}$and $\mathrm{Z}$, and the pseudoscalar component of $\mathrm{S}$ in $Z^{\prime}$, we are left with only two scalar Higgs, $h^{0}$ and $s^{0}$. The squared mass matrix in the $\left(h^{0}, s^{0}\right)$ basis is given by

$$
\mathcal{M}^{2}=2 v^{2}\left(\begin{array}{cc}
2 \lambda_{H} & \lambda_{H S} \alpha \\
\lambda_{H S} \alpha & 2 \lambda_{S} \alpha^{2}
\end{array}\right)
$$

where $\alpha=v_{s} / v$.

The mass eigenstates $h$ and $s$ can be written as

$$
\begin{aligned}
h^{0} & =h \cos \theta+s \sin \theta, \\
s^{0} & =-h \sin \theta+s \cos \theta,
\end{aligned}
$$

where $\theta$ is the mixing angle in the Higgs sector.

In the Yukawa interactions discussed above, as well as in the gauge interactions involving the Higgs fields, the fields appearing are $h^{0}$ and $s^{0}$, and these can be expressed in terms of $h$ and $s$ using eq. (12).

The mass of the $Z^{\prime}$ gauge boson is given by

$$
m_{Z^{\prime}}^{2}=2 g_{E}^{2} v_{s}^{2}
$$

Note that the $Z^{\prime}$ does not couple to any SM particles directly. The $Z^{\prime}$ coupling to the SM particles will be only via dimension six or higher operators. Such couplings will be generated by the vectorlike fermions in the model to be discussed in section 5 .

\section{Phenomenological Implications: Constraints from existing data}

In this section, we discuss the constraints on our model from the existing experimental results. As can be seen from eq. (9), the exchange of $s$ gives rise to tree level FCNC processes. This will cause $K^{0}-\bar{K}^{0}$ mass splitting, $D^{0}-\bar{D}^{0}$ mixing, $K_{L} \rightarrow \mu^{+} \mu^{-}, B_{s}^{0} \rightarrow \mu^{+} \mu^{-}$, as well as contributions to the electric dipole moment $(\mathrm{EDM})$ of neutron and electron, and other rare processes that we discuss below. 


\section{$3.1 \quad \mathrm{~K}^{0}-\overline{\mathrm{K}}^{0}$ mixing}

In our model, this arises from the tree level $s$ exchange between $d \bar{s}$ and $\bar{s} d$, and is proportional to $\beta^{2} \epsilon^{10}$. Taking $\beta \sim \epsilon \sim 0.15$, and the values of the couplings $h_{12}^{d}$ and $h_{21}^{d}$ to be of order 1 , the contribution to $\Delta m_{K}^{\mathrm{Higgs}} \simeq 10^{-16}$ to $10^{-17} \mathrm{GeV}$, for an $s$ mass of $100 \mathrm{GeV}$. The experimental value of $\Delta m_{K}$ is $3.5 \times 10^{-15}$ $\mathrm{GeV}$ [9]. Thus, since the contribution goes like $m_{s}^{-4}$, s can be lighter than $100 \mathrm{GeV}$. Note that $\epsilon=v_{s} / M$ is fixed to be $\sim 0.15$ to explain fermion mass hierarchy and the CKM mixing. However, $\beta=v / M$ is a parameter in our model. Although the $\Delta m_{K}$ constraint allows a somewhat larger value of $\beta$, we shall see that $D^{0}-\bar{D}^{0}$ mixing constrains $\beta \sim \epsilon$.

\section{$3.2 \quad \mathrm{D}^{0}-\overline{\mathrm{D}}^{0}$ mixing}

This contribution is again due to the tree level $s$ exchange between $u \bar{c}$ and $\bar{u} c$, and is proportional to $\beta^{2} \epsilon^{6}$, and hence is enhanced compared to $\Delta m_{K}$. Again, taking the couplings $h_{12}^{u}$ and $h_{21}^{u}$ to be of order one and $\beta \sim \epsilon$, we get $\Delta m_{D} \sim 10^{-14} \mathrm{GeV}$ for $m_{s}=100 \mathrm{GeV}$. This is to be compared with the current experimental value of $1.6 \times 10^{-14} \mathrm{GeV}\left[9\right.$, 10]. Thus $\Delta m_{D}$ gives a much stronger restriction on the model parameters. $\beta$ can not be much larger than $\epsilon$, and $s$ can not be much lighter than $100 \mathrm{GeV}$. If our proposal is correct, an electroweak singlet scalar should be observed at the LHC.

\subsection{Other rare processes}

In our model, tree level $s$ exchange between $d \bar{s}$ and $\mu^{+} \mu^{-}$will contribute to $K_{L} \rightarrow \mu^{+} \mu^{-}$. This contribution is proportional to $\beta^{2} \epsilon^{10}$, and leads to a contribution to this branching ratio $\sim 10^{-14}$ for $\beta \sim \epsilon$ and $m_{s} \sim 100$ $\mathrm{GeV}$. This is very small compared to the current experimental value of $\sim 6.9 \times 10^{-9}$ [9. Similarly, the contribution to the other rare processes such as $K_{L} \rightarrow \mu e, K \rightarrow \pi \bar{\nu} \nu, \mu \rightarrow e \gamma, \mu \rightarrow 3 e, B_{d}-\overline{B_{d}}$ mixing, etc are several orders of magnitude below the corresponding experimental limits.

\subsection{Constraint on the mass of $s$}

Experiments at LEP2 have set a lower limit of $114.4 \mathrm{GeV}$ for the mass of the SM Higgs boson, from nonobservation of the associated production $e^{+} e^{-} \rightarrow Z h$. In our model, since the singlet Higgs can mix with the doublet $h$, there will be a limit for $m_{s}$ depending on the value of the mixing angle, $\theta$. For $\sin ^{2} \theta \geq 0.25$, the bound of 114.4 applies also for $m_{s}$ [11. However, $s$ can be lighter if the mixing is small.

\subsection{Constraint on the mass of the $Z^{\prime}$}

We have assumed that the extra $U(1)$ symmetry in our model is spontaneously broken at the EW scale. But the corresponding gauge coupling, $g_{E}$ is arbitrary and hence the mass of $Z^{\prime}$ is not determined in our model. However, very accurately measured $Z$ properties at LEP1 put a constraint on the $Z-Z^{\prime}$ mixing to be $\sim 10^{-3}$ or smaller $\left[9,12\right.$. In our model, the $Z^{\prime}$ does not couple to any SM particle directly. $Z-Z^{\prime}$ mixing can take place at the one loop level with the new vectorlike fermions in the loop. The mixing angle is

$$
\theta_{Z Z^{\prime}} \sim \frac{g_{Z} g_{E}}{16 \pi^{2}}\left(\frac{m_{Z}}{M}\right)^{2}
$$


where $\mathrm{M}$ is the mass of the vectorlike fermions with masses in the TeV scale. Even with $g_{E} \sim 1$, we get $\theta_{Z Z^{\prime}} \sim 10^{-4}$ or less. Thus there is no significant bound for the mass of this $Z^{\prime}$ from the LEP1. This $Z^{\prime}$ can couple to the SM particles via dimension six operators with the interaction of the form

$$
L=\frac{\bar{\psi}_{L} \sigma^{\mu \nu} \psi_{R} H Z^{\prime \mu \nu}}{M^{2}} .
$$

As was shown in [13, no significant bound on $m_{Z^{\prime}}$ emerges from these interactions.

\section{Phenomenological Implications: New physics signals}

Motivated to explain the observed mass hierarchy in the fermion sector, we have constructed a model which has a complex singlet Higgs (in addition to the usual doublet), a new $U(1)_{S}$ gauge symmetry at the EW scale, and a new set of vectorlike fermions at the TeV scale. Thus our model has new particles such as a scalar Higgs and a new $Z^{\prime}$ boson at the EW scale, and heavy vectorlike quarks and leptons. The model has many phenomenological implications for the production and decays of the Higgs bosons, top quark physics, a new scenario for $Z^{\prime}$ physics, and the production and decays of the vectorlike fermions.

\subsection{Higgs signals}

\subsubsection{Higgs coupling to the SM fermions}

As can be seen from (8), the couplings of the doublet Higgs $h$ to the SM fermions are identical to that in the SM, whereas the couplings of the singlet Higgs have a different flavor dependence. In particular, the singlet Higgs $s$ does not couple to the top quark, whereas its couplings to $(b, \tau ; c, s, \mu ; u, d, e)$ involve the flavor dependent factors $(2,2 ; 2,4,4 ; 6,6,6)$ respectively, in the limit of zero mixing between $h$ and $s$. Including the mixing, these factors will be modified. Thus our model will be distinguished from the SM by the fact that

the Higgs has nonstandard couplings to fermions predicted in terms of two model parameters: the ratio of vevs $\alpha$ and the mixing angle $\theta$.

\subsubsection{Higgs decays}

The couplings of the Higgs bosons $h$ and $s$ to the fermions and the gauge bosons can be obtained from eqns. (8) and (9), and are given in Table 1.

The coupling of $h$ to $s$ given by:

$$
\begin{aligned}
\lambda_{\mathrm{hss}}= & \frac{m_{h}^{2}}{4 v}\left\{(1-\mu) \sin 2 \theta\left[\cos ^{3} \theta-\alpha \sin ^{3} \theta+\sin 2 \theta(\alpha \cos \theta-\sin \theta)\right]+\right. \\
& 3 \sin 2 \theta[\sin \theta(1+\mu-(1-\mu) \cos 2 \theta)-\cos \theta(1+\mu-(1-\mu) \cos 2 \theta) / \alpha]\},
\end{aligned}
$$

where $\mu=m_{s}^{2} / m_{h}^{2}$.

Because of the flavor dependence of the couplings, the branching ratios (BR) for $h$ to various final states are altered substantially from those in the SM. These branching ratios for $h$ to various final states are shown in Figs. 1 14 for values of the mixing angle $\theta=0,20^{\circ}, 26^{\circ}$, and $40^{\circ}$ respectively.

For $\theta=0$, i.e. no mixing, these BR's are the same as for the SM Higgs. Note that for both $\theta=20^{\circ}$ and $26^{\circ}$, the $g g$ and the $\gamma \gamma$ BR's are enhanced substantially compared to the SM. This is due to drastic reduction for the $b \bar{b}$ mode from an approximate cancellation in the corresponding coupling as can be seen from Table 1 . 
Higgs Branching Ratios, $\theta=0$

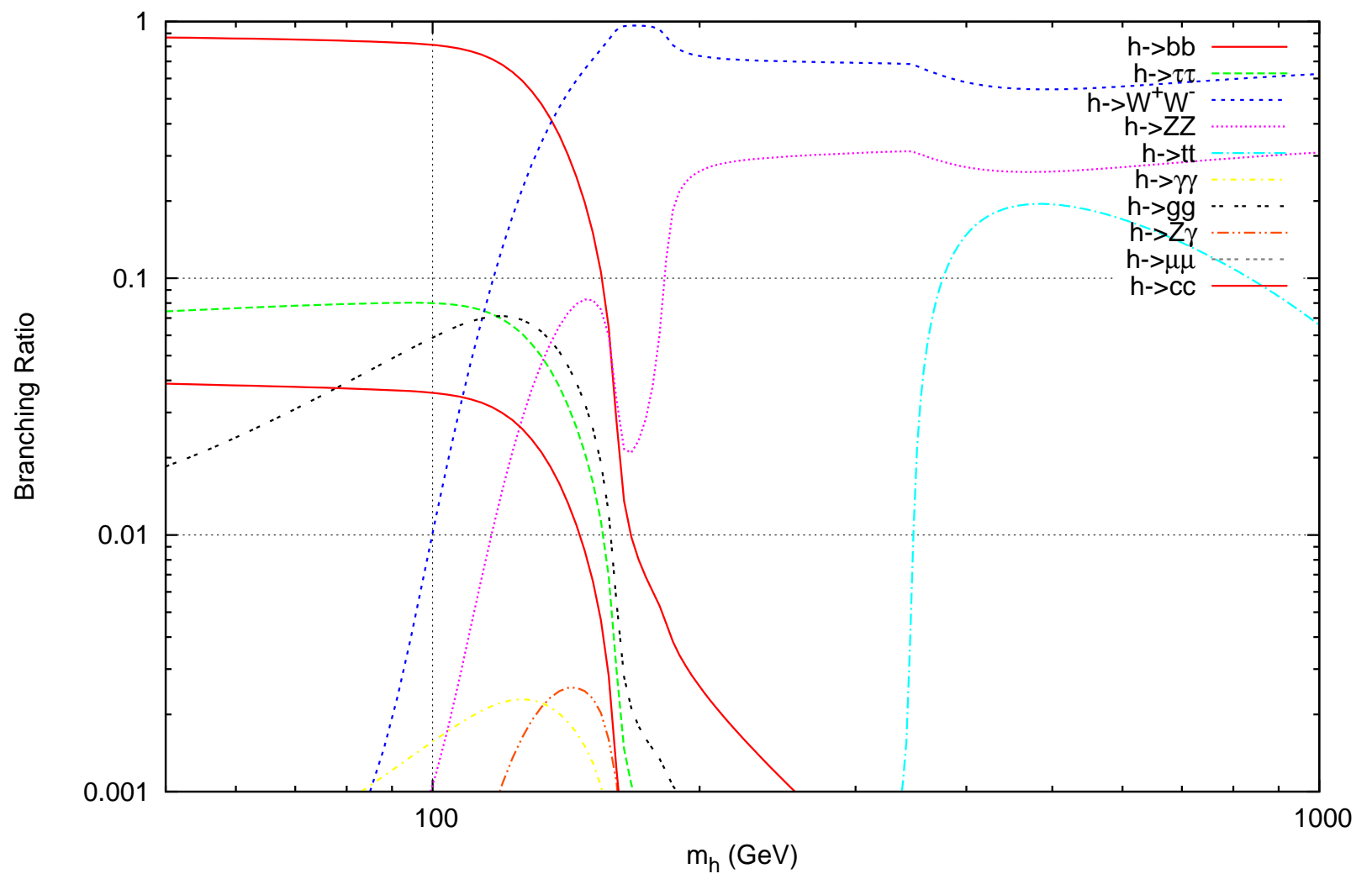

Figure 1: Branching ratio of $h \rightarrow 2 x$, for $\theta=0$ and $\alpha=1[18$. 
Higgs Branching Ratios, $\theta=20$

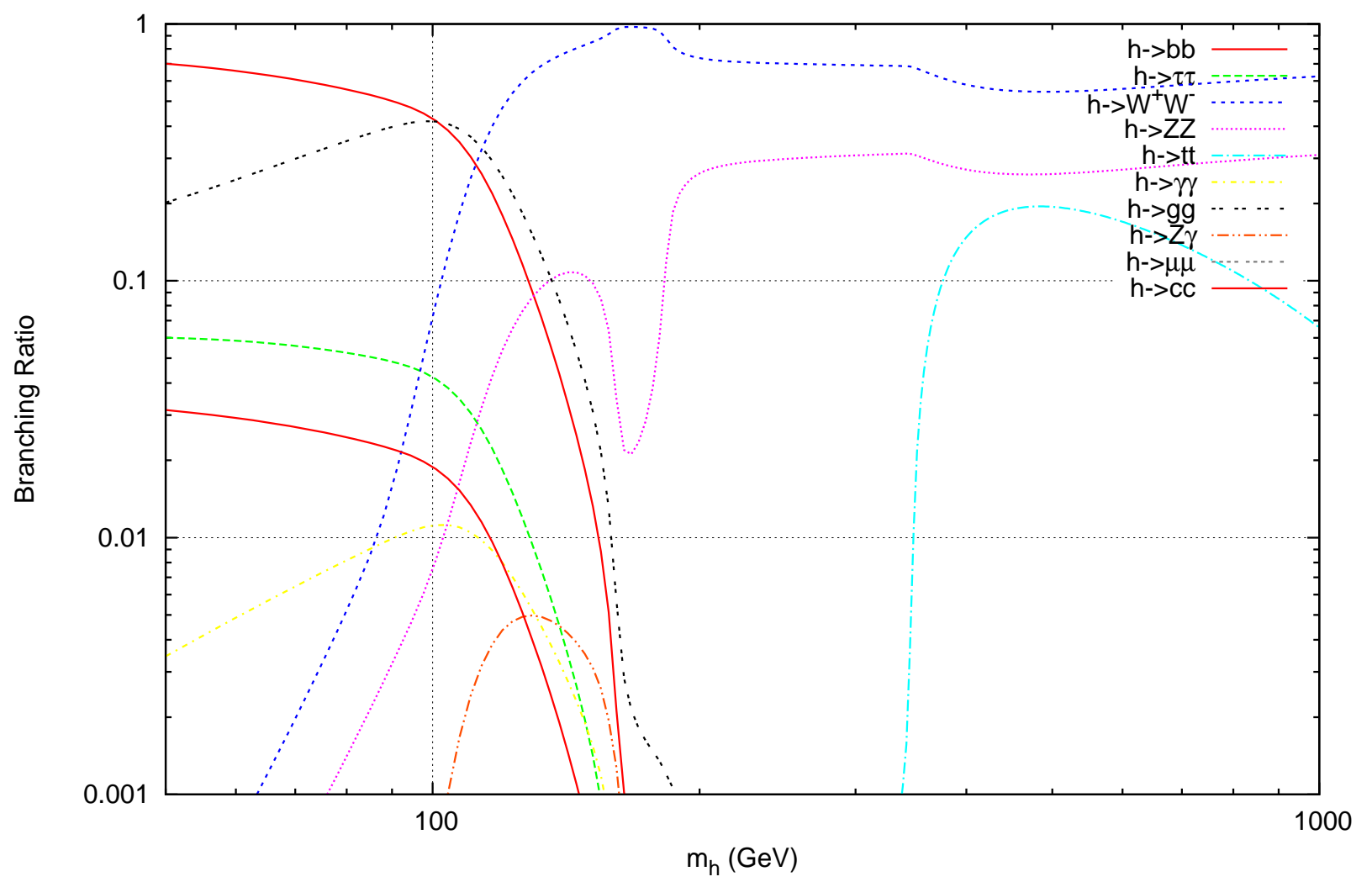

Figure 2: Branching ratio of $h \rightarrow 2 x$, for $\theta=20^{\circ}$ and $\alpha=1$. 


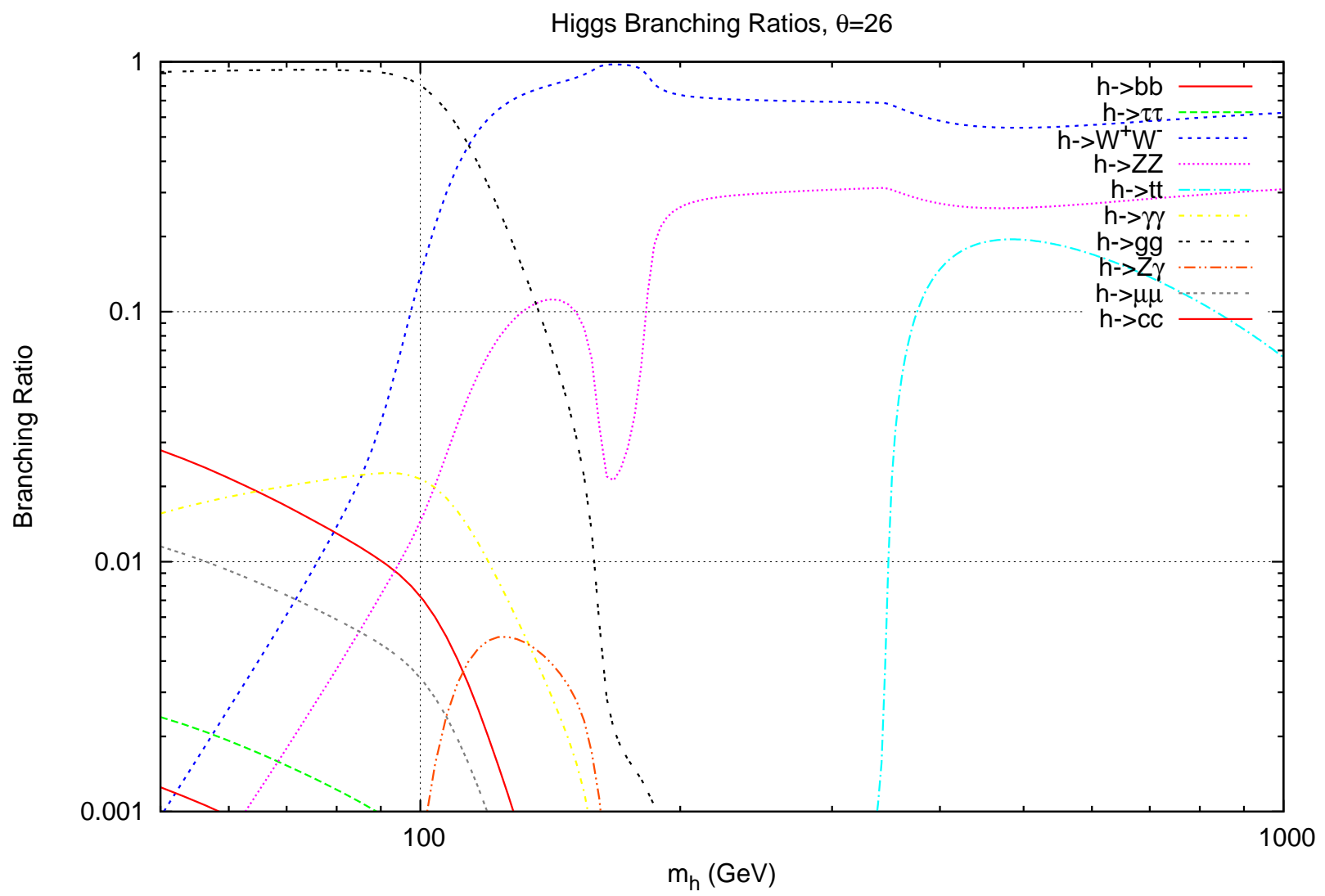

Figure 3: Branching ratio of $h \rightarrow 2 x$, for $\theta=26^{\circ}$ and $\alpha=1$. 


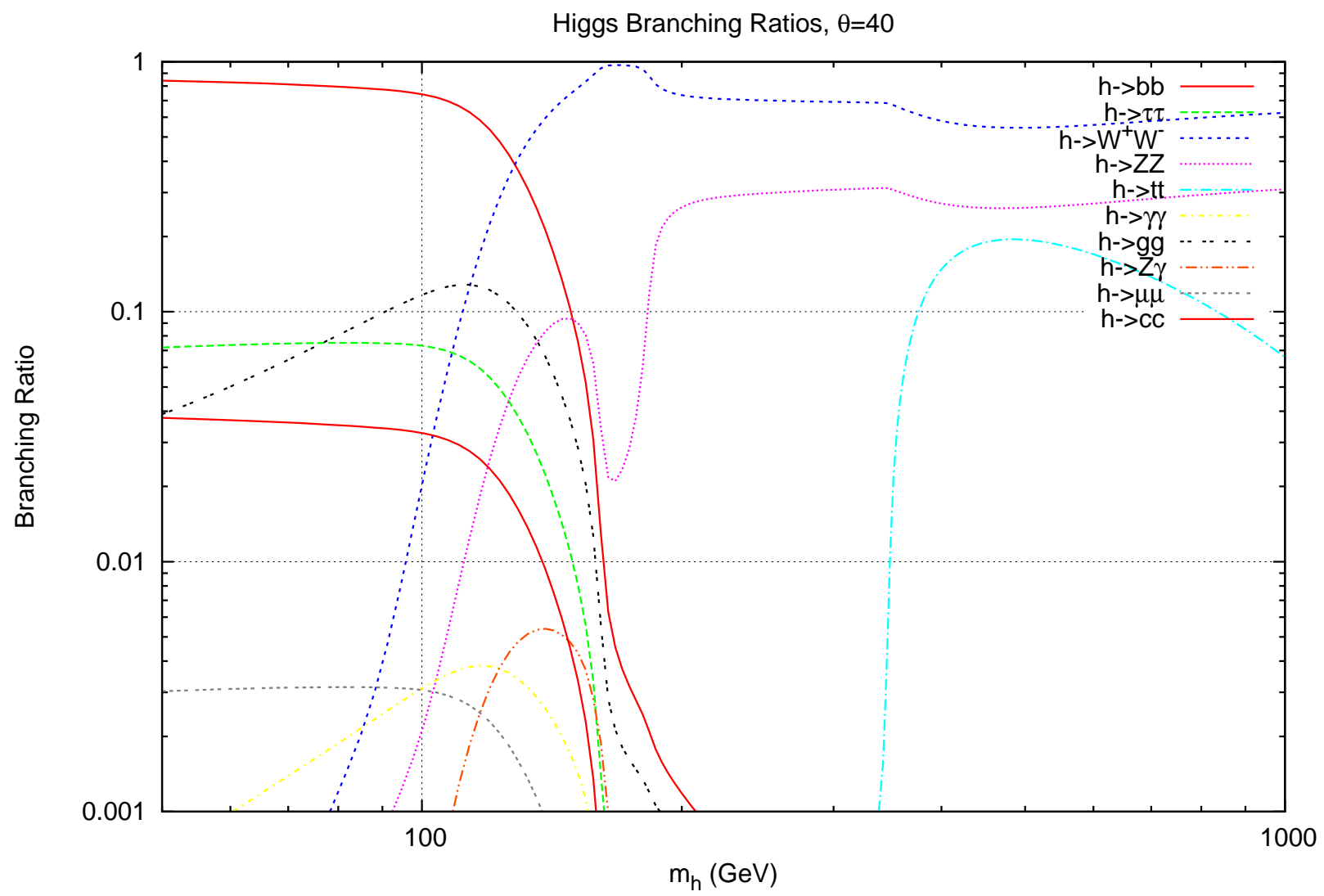

Figure 4: Branching ratio of $h \rightarrow 2 x$, for $\theta=40^{\circ}$ and $\alpha=1$. 


\begin{tabular}{|l|c||l|c|}
\hline Interaction & Coupling & Interaction & Coupling \\
$s \rightarrow u \bar{u}$ & $\frac{m_{u}}{v \sqrt{2}}\left(\sin \theta+\frac{6 \cos \theta}{\alpha}\right)$ & $h \rightarrow u \bar{u}$ & $\frac{m_{u}}{v \sqrt{2}}\left(\cos \theta-\frac{6 \sin \theta}{\alpha}\right)$ \\
$s \rightarrow d \bar{d}$ & $\frac{m_{d}}{v \sqrt{2}}\left(\sin \theta+\frac{6 \cos \theta}{\alpha}\right)$ & $h \rightarrow d \bar{d}$ & $\frac{m_{d}}{v \sqrt{2}}\left(\cos \theta-\frac{6 \sin \theta}{\alpha}\right)$ \\
$s \rightarrow \mu^{+} \mu^{-}$ & $\frac{m_{\mu}}{v \sqrt{2}}\left(\sin \theta+\frac{4 \cos \theta}{\alpha}\right)$ & $h \rightarrow \mu^{+} \mu^{-}$ & $\frac{m_{\mu}}{v \sqrt{2}}\left(\cos \theta-\frac{4 \sin \theta}{\alpha}\right)$ \\
$s \rightarrow s \bar{s}$ & $\frac{m_{s}}{v \sqrt{2}}\left(\sin \theta+\frac{4 \cos \theta}{\alpha}\right)$ & $h \rightarrow s \bar{s}$ & $\frac{m_{s}}{v \sqrt{2}}\left(\cos \theta-\frac{4 \sin \theta}{\alpha}\right)$ \\
$s \rightarrow \tau^{+} \tau^{-}$ & $\frac{m_{\tau}}{v \sqrt{2}}\left(\sin \theta+\frac{2 \cos \theta}{\alpha}\right)$ & $h \rightarrow \tau^{+} \tau^{-}$ & $\frac{m_{\tau}}{v \sqrt{2}}\left(\cos \theta-\frac{2 \sin \theta}{\alpha}\right)$ \\
$s \rightarrow c \bar{c}$ & $\frac{m_{c}}{v \sqrt{2}}\left(\sin \theta+\frac{2 \cos \theta}{\alpha}\right)$ & $h \rightarrow c \bar{c}$ & $\frac{m_{c}}{v \sqrt{2}}\left(\cos \theta-\frac{2 \sin \theta}{\alpha}\right)$ \\
$s \rightarrow b \bar{b}$ & $\frac{m_{b}}{v \sqrt{2}}\left(\sin \theta+\frac{2 \cos \theta}{\alpha}\right)$ & $h \rightarrow b \bar{b}$ & $\frac{m_{b}}{v \sqrt{2}}\left(\cos \theta-\frac{2 \sin \theta}{\alpha}\right)$ \\
$s \rightarrow t \bar{t}$ & $\frac{m_{t}}{v \sqrt{2}} \sin \theta$ & $h \rightarrow t \bar{t}$ & $\frac{m_{t}}{v \sqrt{2}} \cos \theta$ \\
$s \rightarrow Z Z$ & $\frac{2 m_{Z}^{2}}{v \sqrt{2}} \sin \theta$ & $h \rightarrow Z Z$ & $\frac{2 m_{Z}^{2}}{v \sqrt{2}} \cos \theta$ \\
$s \rightarrow Z^{\prime} Z^{\prime}$ & $\frac{m_{Z^{\prime}}^{2}}{v \alpha \sqrt{2}} \cos \theta$ & $h \rightarrow Z^{\prime} Z^{\prime}$ & $\frac{m_{Z^{\prime}}^{2}}{v \alpha \sqrt{2}} \sin \theta$ \\
$s \rightarrow W^{+} W^{-}$ & $\frac{2 m_{W}^{2}}{v \sqrt{2}} \sin \theta$ & $h \rightarrow W^{+} W^{-}$ & $\frac{2 m_{W}^{2}}{v \sqrt{2}} \cos \theta$ \\
\hline
\end{tabular}

Table 1: Yukawa and gauge couplings of $h$ and $s$.

In particular, for $\theta=26^{\circ}$, the effect is quite dramatic. For a light Higgs $\left(m_{h}\right.$ around $\left.115 \mathrm{GeV}\right)$, the usually dominant $b \bar{b}$ mode is highly suppressed and the $\gamma \gamma$ mode is enhanced by a factor of almost 10 compared to the SM. This is to be contrasted with the proposal of Refs. 6, 7] in which the $h \rightarrow \gamma \gamma$ mode is reduced by about a factor of 10. Thus the Higgs signal in this mode for a Higgs mass of $\sim 114-140 \mathrm{GeV}$ gets a big enhancement, making its potential discovery via this mode much more favorable at the LHC. Such a signal may be observable at the Tevatron for a Higgs mass $\sim 114$ as the luminosity accumulates, but would require about $10 \mathrm{fb}^{-1}$ or more of data $[14$.

Another interesting effect is the Higgs signal via the $W W^{*}$ for the light Higgs. In the SM, this mode becomes important for the Tevatron search for Higgs masses greater than about $135 \mathrm{GeV}$, where the BR to $W W^{*}$ is approximate equal to that of $b \bar{b}$. Currently Tevatron experiments have excluded a SM Higgs with mass around $170 \mathrm{GeV}$ (where the BR to $W W^{*}$ is around 100 percent) for this mode [15. In our framework, for $\theta=20^{\circ}$ for example, the crossover between the $W W^{*}$ mode and the $b \bar{b}$ mode takes place sooner than 135 GeV. Thus the Tevatron experiments will be more sensitive to the lower mass range than for a SM Higgs, and should be able to exclude masses much smaller than $160 \mathrm{GeV}$.

For a heavy Higgs, $m_{h}>200 \mathrm{GeV}$, the Higgs will be accessible via the golden mode $h \rightarrow Z Z$. However, in this case, both $h$ and $s$ decay via this mode with comparable BR's (see Figs. 2 and 4 for $\theta=20^{\circ}$ and $40^{\circ}$ ). So initially it will be hard to tell whether we are seeing $h$ or $s$, a case of Higgs look-alikes. An accurate measurement of this cross section times the BR, and the mass of the observed Higgs, we will be able to distinguish a heavy $h$ from a heavy $s$, since the production cross sections depend on the mixing angle.

\subsection{Top quark physics}

In the SM, the $t \rightarrow c h$ mode is severely suppressed with a $\mathrm{BR} \sim 10^{-14}[16$. In our model, as can be seen from eqs. (8) and (9), although $t \rightarrow c h$ is zero at tree level, we have a large coupling for $t \rightarrow c s \sim 2 \epsilon \beta$ (note $s$ here denotes the singlet Higgs, not the strange quark). This gives rise to a significant BR for the $t \rightarrow c s$ mode for a Higgs mass of up to about $150 \mathrm{GeV}$. If the mixing between the $\mathrm{h}$ and $\mathrm{s}$ is substantial, both decay 
modes, $t \rightarrow c s$ and $t \rightarrow c h$ will have $\mathrm{BR} \sim 10^{-3}$. With a very large $t \bar{t}$ cross section, $\sigma_{t \bar{t}} \sim 10^{3} \mathrm{pb}$ at the LHC, this could be an observable production mode for Higgs bosons at the LHC.

\section{3 $\quad \mathrm{Z}^{\prime}$ physics}

Our model has a $Z^{\prime}$ boson near the EW scale from the spontaneous breaking of the extra $U(1)$ symmetry. As discussed before, since the $Z-Z^{\prime}$ mixing is very small $\sim 10^{-4}$ or less, its mass is not constrained by the very accurately measured $Z$ properties at LEP. Its mass can be as low as a few GeV from the existing constraints. This $Z^{\prime}$ does not couple to SM particles with dimension 4 operators. It does couple to $s$ at tree level via the $s Z^{\prime} Z^{\prime}$ interaction. Thus it can be produced via the decay of $s$ (or $h$ if there is a substantial mixing between $h$ and $s$ ). This gives an interesting signal for the Higgs decays: $s \rightarrow Z^{\prime} Z^{\prime}, h \rightarrow Z^{\prime} Z^{\prime}$ if allowed kinematically. In Figs. 5 and 6 , we give the BR's for $h$ and $s$ decays for a $Z^{\prime}$ mass of $40 \mathrm{GeV}$. The $Z^{\prime}$ will decay to the SM particles via the $Z-Z^{\prime}$ mixing with the same branching ratio as the $Z$. Thus the clear final state signal will be $l^{+} l^{-} l^{+} l^{-}$pairs $(l=e, \mu)$ with each pair having the invariant mass of the $Z^{\prime}$. Such a signal will be easily detectable at the LHC. If the $Z^{\prime}$ happens to be very light, (say a few GeV), and the mixing angle is extremely tiny, there is a possibility that the $Z^{\prime}$ s may produce displaced vertices at the detector. Both of these will be very unconventional signals for Higgs bosons at the LHC.

\section{$4.4 \quad \mathbf{B}_{\mathbf{s}}^{0} \rightarrow \mu^{+} \mu^{-}$}

In our framework this decay gets a contribution from an FCNC interaction mediated by $s$ exchange. The amplitude for this decay is $A \sim 4 h_{22}^{d} h_{22}^{\ell} \epsilon^{6} \beta^{2}$. Taking $\beta \sim \epsilon, A \sim 4 h_{22}^{d} h_{22}^{\ell} \epsilon^{8}$, and with the couplings $h_{22}^{d}, h_{22}^{\ell} \sim 1$, we obtain the branching ratio, $B R\left(B_{s}^{0} \rightarrow \mu^{+} \mu^{-}\right) \sim 10^{-9}$. The current experimental limit for this BR is $4.7 \times 10^{-8}[9$, and thus there is a possibility that this decay could be observed at the Tevatron.

\subsection{Production and decay of heavy fermions}

Our framework requires vectorlike quarks and leptons, both $S U(2)$ doublets and weak singlets, with masses around the TeV scale. The heavy quarks be pair produced at high energy hadron colliders via the strong interaction. For example, for a $1 \mathrm{TeV}$ vectorlike quark, the production cross section at the LHC is $\sim 60 \mathrm{fb}$ 17. We need several such vectorlike quarks for our model. So the total production cross section could be as large as a few hundred fb. These will decay to the light quarks of the same electric charge and Higgs bosons $\left(h\right.$ or $s$ ): Thus the signal will be two high $p_{T}$ jets together with the final states arising from the Higgs decays. For a heavy Higgs, in the golden mode $\left(h \rightarrow Z Z, s \rightarrow Z Z\right.$, this will give rise to two high $p_{T}$ jets plus four $Z$ bosons. In the case of a light $Z^{\prime}$, the final state signal will be two high $p_{T}$ jets plus up to 8 charged leptons in the final state (with each lepton pair having the invariant mass of the $Z^{\prime}$ ).

\section{UV Completion}

We present two concrete examples of models from which an effective action like eq. (1) can be derived. The first example only reproduces the second and third generation quark couplings, but its simplicity serves to introduce the basic issues and mechanisms. The second example is a complete three generation TeV scale model of quark flavor. The correct lepton couplings can be obtained from a copy of the same structure used for the down-type quarks. We assume that neutrino masses benefit from some additional see-saw mechanism, although it is not obvious that we can't obtain them by refining the $\mathrm{TeV}$ scale flavon model. 
Higgs Branching Ratios in Model, $\theta=20$

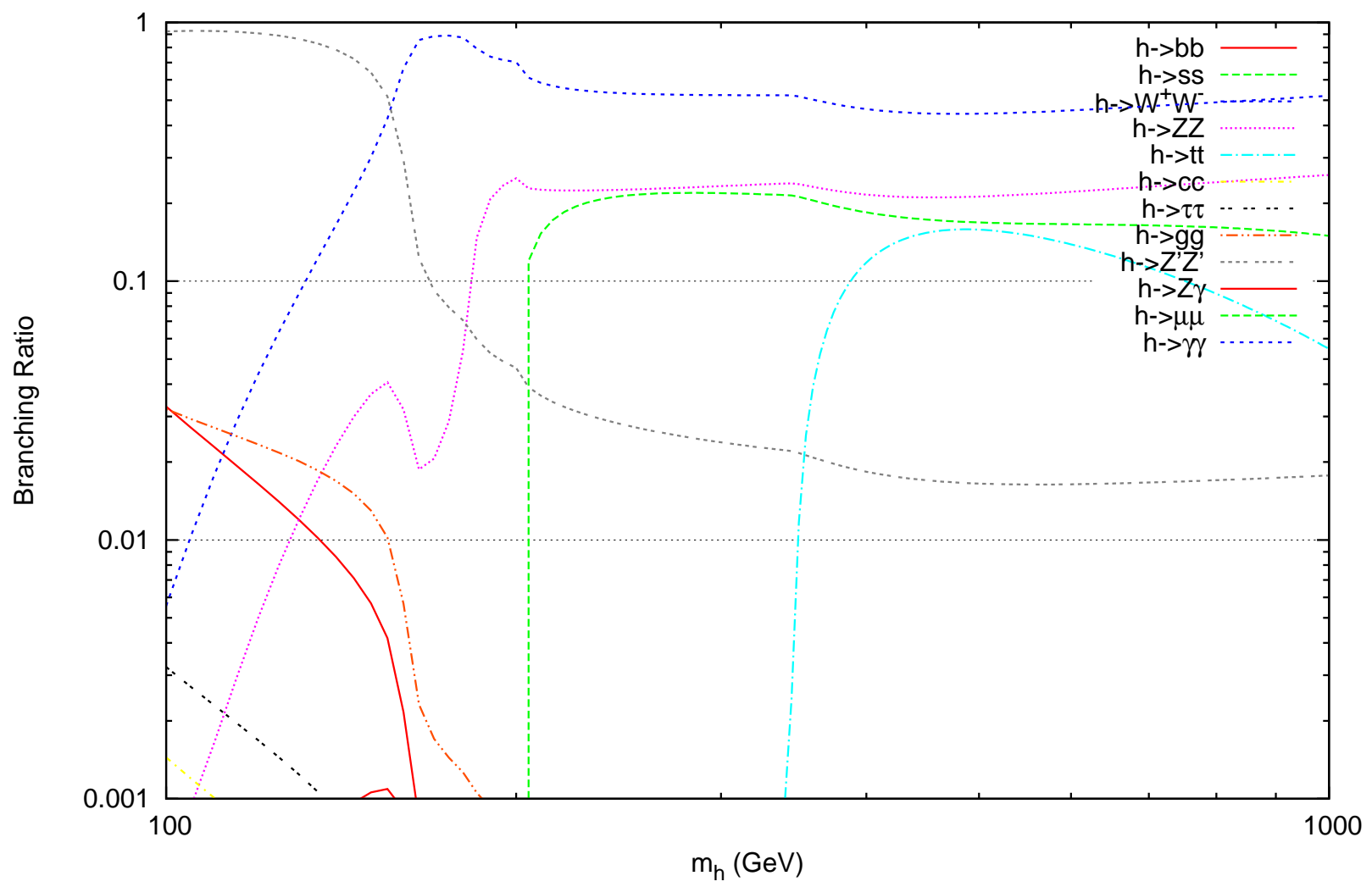

Figure 5: Branching ratio of $h \rightarrow 2 x$ including $h \rightarrow s s$ and $h \rightarrow Z^{\prime} Z^{\prime}$ where $m_{Z^{\prime}}=40 \mathrm{GeV}$ and $m_{s}=100$ GeV. Here $\theta=20^{\circ}$ and $\alpha=1$. 


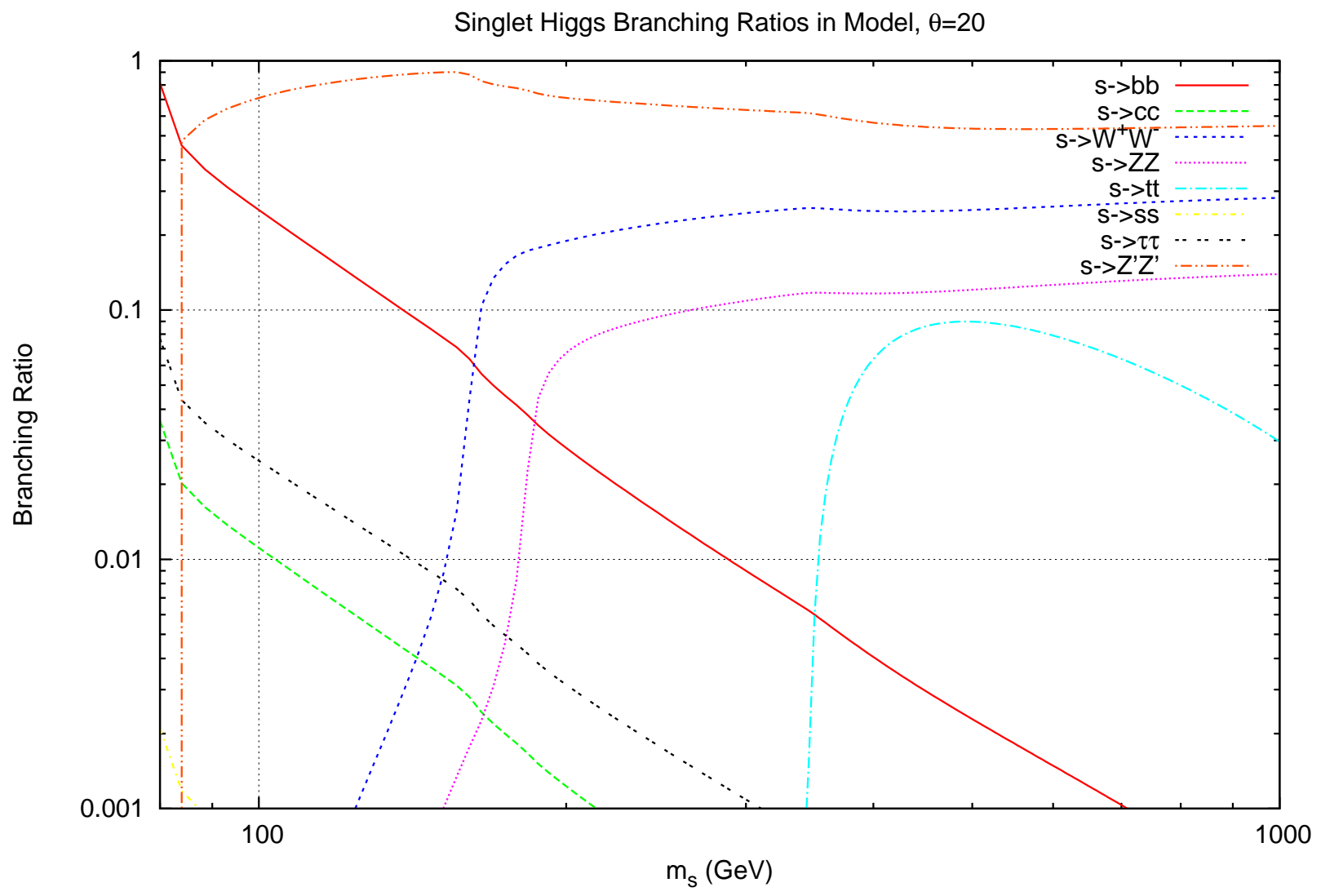

Figure 6: Branching ratio of $s \rightarrow 2 x$ including $s \rightarrow Z^{\prime} Z^{\prime}$ where $m_{Z^{\prime}}=40 \mathrm{GeV}$. Here $\theta=20^{\circ}$ and $\alpha=1$. 


\begin{tabular}{|c|c|c|c||c|c|c|c|}
\hline Field & $\mathbf{U}(\mathbf{1})_{\mathbf{Y}}$ & $\mathbf{U}(\mathbf{1})_{\mathbf{S}}$ & $\mathbf{U}(\mathbf{1})_{\mathbf{F}}$ & Field & $\mathbf{U}(\mathbf{1})_{\mathbf{Y}}$ & $\mathbf{U}(\mathbf{1})_{\mathbf{S}}$ & $\mathbf{U}(\mathbf{1})_{\mathbf{F}}$ \\
\hline$H$ & $1 / 2$ & 0 & 0 & $U_{1 L}$ & $2 / 3$ & 1 & 0 \\
$S$ & 0 & 1 & 0 & $U_{1 R}$ & $2 / 3$ & 1 & 1 \\
$F$ & 0 & 0 & 1 & $U_{2 L}$ & $2 / 3$ & -1 & 3 \\
$q_{3 L}$ & $1 / 6$ & 0 & 0 & $U_{2 R}$ & $2 / 3$ & -1 & 3 \\
$q_{2 L}$ & $1 / 6$ & 0 & 2 & $D_{1 L}$ & $-1 / 3$ & -1 & -1 \\
$u_{3 R}$ & $2 / 3$ & 0 & 0 & $D_{1 R}$ & $-1 / 3$ & -1 & -1 \\
$u_{2 R}$ & $2 / 3$ & 0 & 3 & $D_{2 L}$ & $-1 / 3$ & 2 & 3 \\
$d_{3 R}$ & $-1 / 3$ & 0 & -1 & $D_{2 R}$ & $-1 / 3$ & 2 & 2 \\
$d_{2 R}$ & $-1 / 3$ & 0 & 3 & $D_{3 L}$ & $-1 / 3$ & 1 & 3 \\
$Q_{1 L}$ & $1 / 6$ & -1 & -1 & $D_{3 R}$ & $-1 / 3$ & 1 & 3 \\
$Q_{1 R}$ & $1 / 6$ & -1 & 0 & & & & \\
$Q_{2 L}$ & $1 / 6$ & 1 & 1 & & & & \\
$Q_{2 R}$ & $1 / 6$ & 1 & 2 & & & & \\
$Q_{3 L}$ & $1 / 6$ & -1 & 3 & & & & \\
$Q_{3 R}$ & $1 / 6$ & -1 & 2 & & & & \\
$Q_{4 L}$ & $1 / 6$ & 2 & 2 & & & & \\
$Q_{4 R}$ & $1 / 6$ & 2 & 1 & & & & \\
\hline
\end{tabular}

Table 2: Charge assignments in the two generation model for the scalar fields $H, S, F$, and the SM quark fields $q_{3 L}, q_{2 L}, u_{3 R}, u_{2 R}, d_{3 R}$, and $d_{2 R}$. Also listed are the color triplet weak doublet heavy quark pairs $Q_{i L}$, $Q_{i R}$ and the color triplet weak singlet heavy quark pairs $U_{i L}, U_{i R}, D_{i L}, D_{i R}$.

\subsection{Two generation model}

For this pedagogical example we will employ two important simplifications:

- We only reproduce the second and third generation quark couplings. In the next subsection we extend this to include the first generation.

- We will choose charge assignments such that the couplings $h_{32}^{u}, h_{32}^{d}$, and $h_{23}^{d}$ are higher order in $\epsilon$. As already mentioned nonzero values of these couplings are not needed to reproduce the observed SM quark masses and mixings.

With these simplifications we postulate a TeV scale model with the field content shown in Table 2, where the hypercharges are listed along with the charge assignments under $U(1)_{S}$ and $U(1)_{F}$. The Higgs doublet $H$ is the only scalar that carries hypercharge, while the SM singlet $S$ is the only scalar carrying $U(1)_{S}$ charge. The SM singlet flavon $F$ is the only scalar carrying $U(1)_{F}$ charge. The SM quarks are neutral under $U(1)_{S}$. The third generation up-type quark fields also carry no $U(1)_{F}$ charge, while the other quark fields have flavor-dependent nonzero $U(1)_{F}$ charges.

We introduce four pairs of new color triplet weak doublet fermion fields $Q_{i L}, Q_{i R}$, two pairs of color triplet up-type weak singlets $U_{i L}, U_{i R}$, and three pairs of color triplet down-type weak singlets $D_{i L}, D_{i R}$. Each pair is vectorlike with respect to the SM gauge group and $U(1)_{S}$, thus no anomalies are introduced with respect to these gauge groups, and each vectorlike pair naturally acquires a Dirac mass of order $M$ (when they have the same $U(1)_{F}$ charge) or of order the vev of $F$ (when their $U(1)_{F}$ charges differ by one). We assume that both the vev of $F$ and $M$ are greater than, but of order of, a TeV. Any residual anomaly 
in $U(1)_{F}$ can be handled either by introducing more heavy fermions or using the Green-Schwarz mechanism above the $\mathrm{TeV}$ scale.

With these charge assignments the only dimension 4 couplings involving the second and third generation SM quarks are:

$$
\begin{aligned}
& f_{1} \bar{q}_{3 L} u_{3 R} \bar{H}+f_{2} \bar{q}_{3 L} Q_{1 R} S+f_{3} \bar{D}_{1 L} d_{3 R} S^{\dagger}+f_{4} \bar{q}_{2 L} Q_{2 R} S^{\dagger} \\
& +f_{5} \bar{U}_{1 L} u_{3 R} S+f_{6} \bar{q}_{2 L} Q_{3 R} S+f_{7} \bar{U}_{2 L} u_{2 R} S^{\dagger}+f_{8} \bar{D}_{3 L} d_{2 R} S+\text { h.c. }
\end{aligned}
$$

where the $f_{i}$ are dimensionless coupling constants. Thus the top quark receives the correct mass from electroweak symmetry breaking for $\left|f_{1}\right| \simeq 1$. The other couplings involve the $S$ scalar, but not the Higgs $H$ or the flavon $F$. Both electroweak symmetry breaking and flavor symmetry breaking are communicated to the rest of the SM quark sector via a Froggart-Nielsen type mechanism, integrating out the heavy TeV scale fermions from tree level diagrams that connect SM quark left doublets to SM quark right singlets and to $H$ or $\bar{H}$.

The renormalizable couplings involving just the heavy fermions are:

$$
\begin{aligned}
& f_{9} \bar{Q}_{1 R} Q_{1 L} F+f_{10} \bar{Q}_{1 L} D_{1 R} H+M \bar{D}_{1 R} D_{1 L} \\
& +f_{11} \bar{Q}_{2 R} Q_{2 L} F+f_{12} \bar{Q}_{2 L} U_{1 R} \bar{H}+f_{13} \bar{U}_{1 R} U_{1 L} F \\
& +f_{14} \bar{Q}_{3 R} Q_{3 L} F^{\dagger}+f_{15} \bar{Q}_{3 L} U_{2 R} \bar{H}+M \bar{U}_{2 R} U_{2 L} \\
& +f_{16} \bar{Q}_{2 L} Q_{4 R} S^{\dagger}+f_{17} \bar{Q}_{4 L} Q_{2 R} S+f_{18} \bar{Q}_{4 R} Q_{4 L} F^{\dagger}+f_{19} \bar{Q}_{4 L} D_{2 R} H \\
& +f_{20} \bar{D}_{2 R} D_{2 L} F^{\dagger}+f_{21} \bar{D}_{2 L} D_{3 R} S+M \bar{D}_{3 L} D_{3 R}+\text { h.c. . }
\end{aligned}
$$

Thus, integrating out the heavy fermions in the tree level diagram composed from the couplings

$$
f_{2} \bar{q}_{3 L} Q_{1 R} S+f_{9} \bar{Q}_{1 R} Q_{1 L} F+f_{10} \bar{Q}_{1 L} D_{1 R} H+M \bar{D}_{1 R} D_{1 L}+f_{3} \bar{D}_{1 L} d_{3 R} S^{\dagger}
$$

produces an effective coupling below the $\mathrm{TeV}$ scale proportional to

$$
f_{2} f_{3} f_{9} f_{10} \frac{F}{M} \frac{S^{\dagger} S}{M^{2}} \bar{q}_{3 L} d_{3 R} H+\text { h.c. . }
$$

Integrating out the heavy fermions in the tree level diagram composed from the couplings

$$
f_{4} \bar{q}_{2 L} Q_{2 R} S^{\dagger}+f_{11} \bar{Q}_{2 R} Q_{2 L} F+f_{12} \bar{Q}_{2 L} U_{1 R} \bar{H}+f_{13} \bar{U}_{1 R} U_{1 L} F+f_{5} \bar{U}_{1 L} u_{3 R} S
$$

produces an effective coupling below the $\mathrm{TeV}$ scale proportional to

$$
f_{4} f_{5} f_{11} f_{12} f_{13} \frac{F^{2}}{M^{2}} \frac{S^{\dagger} S}{M^{2}} \bar{q}_{2 L} u_{3 R} \bar{H}+\text { h.c. . }
$$

Integrating out the heavy fermions in the tree level diagram composed from the couplings

$$
f_{6} \bar{q}_{2 L} Q_{3 R} S+f_{14} \bar{Q}_{3 R} Q_{3 L} F^{\dagger}+f_{15} \bar{Q}_{3 L} U_{2 R} \bar{H}+M \bar{U}_{2 R} U_{2 L}+f_{7} \bar{U}_{2 L} u_{2 R} S^{\dagger}
$$

produces an effective coupling below the $\mathrm{TeV}$ scale proportional to

$$
f_{6} f_{7} f_{14} f_{15} \frac{F^{\dagger}}{M} \frac{S^{\dagger} S}{M^{2}} \bar{q}_{2 L} u_{2 R} \bar{H}+\text { h.c. } .
$$




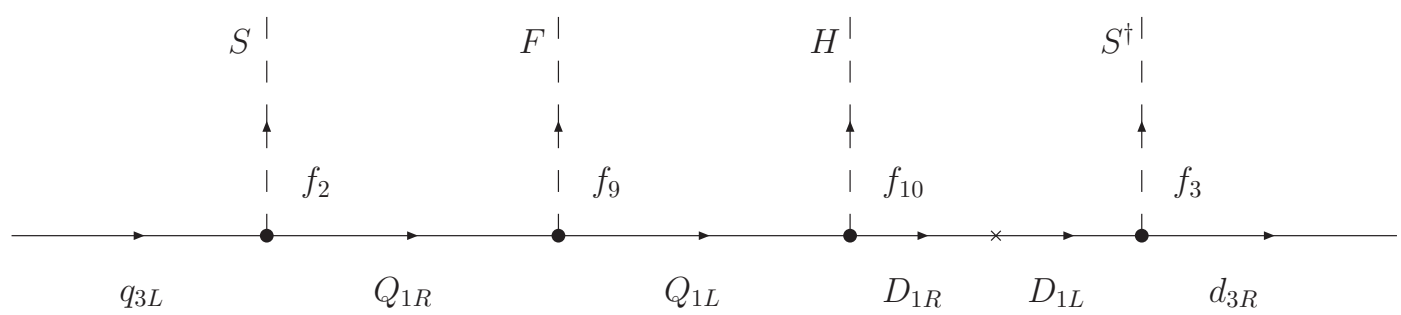

Figure 7: The Feynman diagram associated with eq. (18)

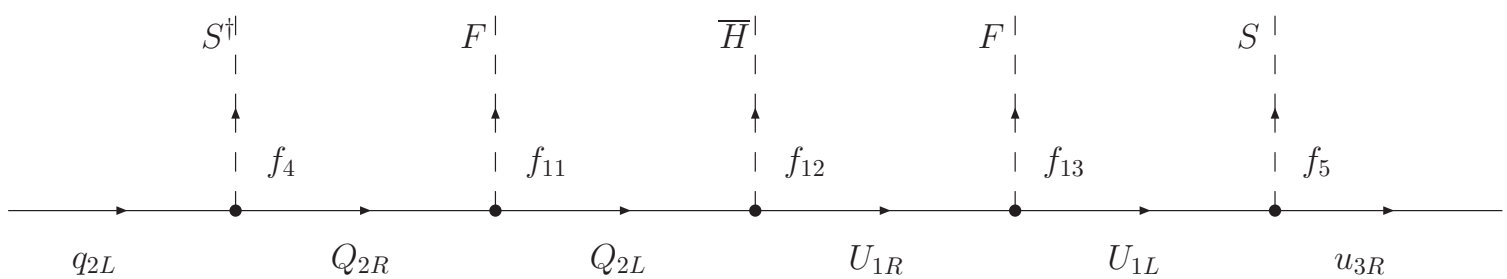

Figure 8: The Feynman diagram associated with eq. (20)

Finally, integrating out the heavy fermions in the tree level diagram composed from the couplings

$$
\begin{aligned}
& f_{4} \bar{q}_{2 L} Q_{2 R} S^{\dagger}+f_{17}^{*} \bar{Q}_{2 R} Q_{4 L} S^{\dagger}+f_{19} \bar{Q}_{4 L} D_{2 R} H \\
& +f_{20} \bar{D}_{2 R} D_{2 L} F^{\dagger}+f_{21} \bar{D}_{2 L} D_{3 R} S+M \bar{D}_{3 R} D_{3 L}+f_{8} \bar{D}_{3 L} d_{2 R} S
\end{aligned}
$$

produces an effective coupling below the $\mathrm{TeV}$ scale proportional to

$$
f_{4} f_{8} f_{17}^{*} f_{19} f_{20} f_{21} \frac{F^{\dagger}}{M} \frac{\left(S^{\dagger} S\right)^{2}}{M^{4}} \bar{q}_{2 L} d_{2 R} H+\text { h.c. . }
$$

There is an additional very similar tree level diagram contributing to $h_{22}^{d}$ composed from the couplings

$$
\begin{aligned}
& f_{4} \bar{q}_{2 L} Q_{2 R} S^{\dagger}+f_{11} \bar{Q}_{2 R} Q_{2 L} F+f_{16} \bar{Q}_{2 L} Q_{4 R} S^{\dagger}+f_{18} \bar{Q}_{4 R} Q_{4 L} F^{\dagger}+f_{19} \bar{Q}_{4 L} D_{2 R} H \\
& +f_{20} \bar{D}_{2 R} D_{2 L} F^{\dagger}+f_{21} \bar{D}_{2 L} D_{3 R} S+M \bar{D}_{3 R} D_{3 L}+f_{8} \bar{D}_{3 L} d_{2 R} S
\end{aligned}
$$

which produces an effective coupling below the $\mathrm{TeV}$ scale proportional to

$$
f_{4} f_{8} f_{11} f_{16} f_{18} f_{19} f_{20} f_{21} \frac{F\left(F^{\dagger}\right)^{2}}{M^{3}} \frac{\left(S^{\dagger} S\right)^{2}}{M^{4}} \bar{q}_{2 L} d_{2 R} H+\text { h.c. . }
$$

\subsection{Three generation model}

Here we present an concrete example of a full three generation $\mathrm{TeV}$ scale model that reproduces an effective action like eq. (11) at the electroweak scale. This model uses a single electroweak messenger scalar $S$, but employs three TeV scale flavon scalars $F_{1}, F_{2}$, and $F_{3}$, each corresponding to a different broken $U(1)_{F_{i}}$ flavon symmetry. As before the SM quarks are neutral under $U(1)_{S}$. The third generation up-type quark fields also carry no $U(1)_{F_{i}}$ charges, while the other quark fields have flavor-dependent nonzero $U(1)_{F_{i}}$ charges. 


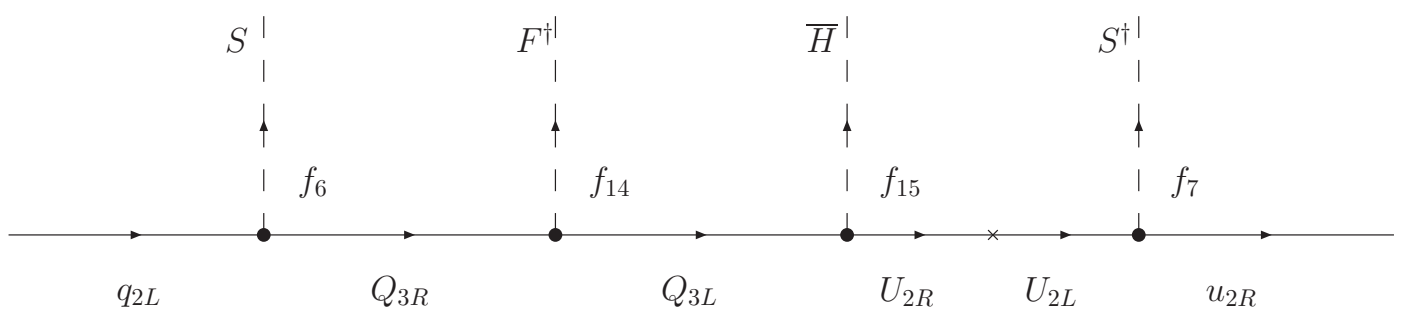

Figure 9: The Feynman diagram associated with eq. (22)
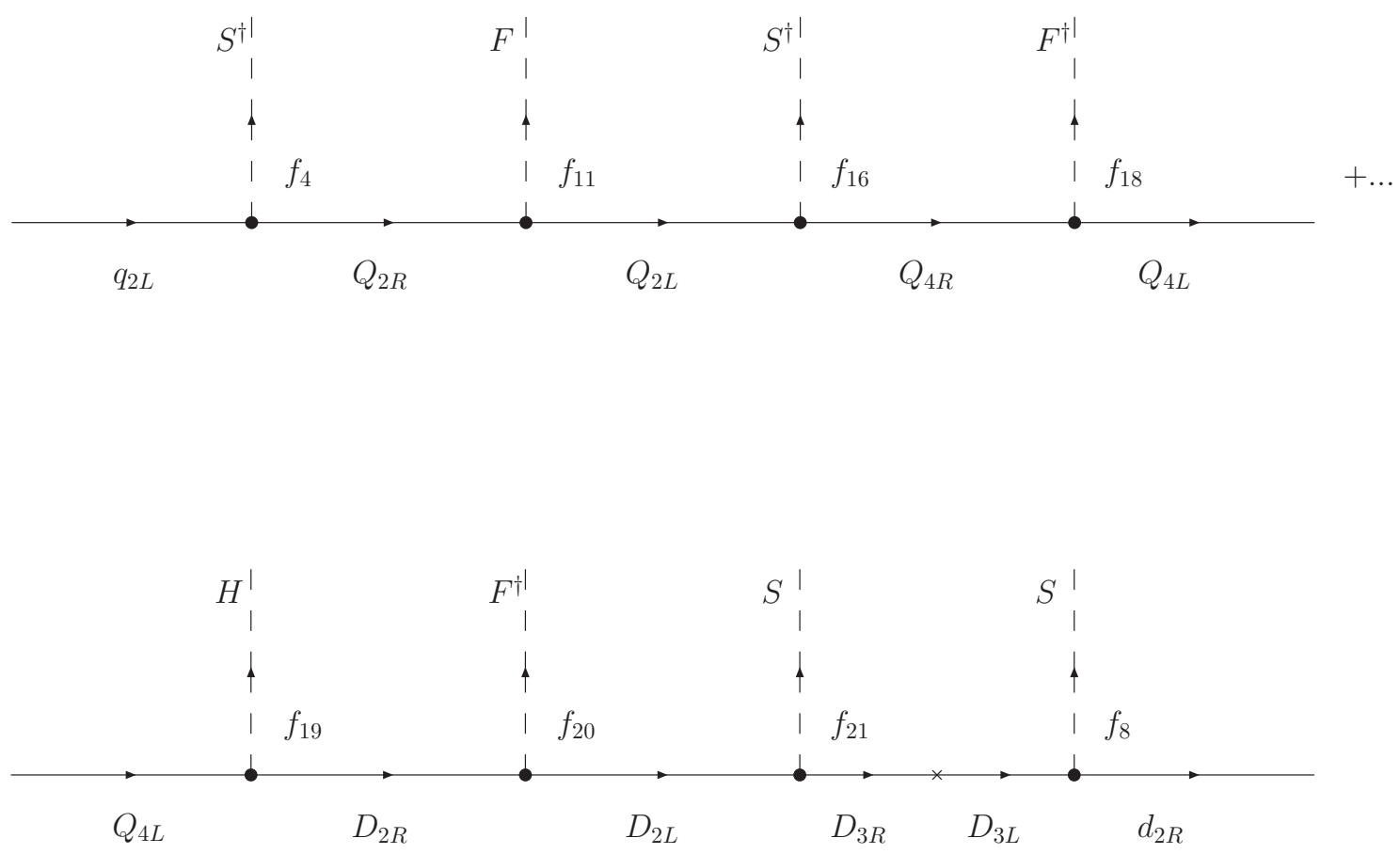

Figure 10: The Feynman diagram associated with eq. (26) 
The model has a rather large number of new heavy fermions: seven pairs of new color triplet weak doublet fermion fields $Q_{i L}, Q_{i R}$, six pairs of color triplet up-type weak singlets $U_{i L}, U_{i R}$, and eight pairs of color triplet down-type weak singlets $D_{i L}, D_{i R}$. Each pair is vectorlike with respect to the SM gauge group and $U(1)_{S}$, thus no anomalies are introduced with respect to these gauge groups, and each vectorlike pair naturally acquires a Dirac mass of order $M$ (when they have the same $U(1)_{F_{i}}$ charges) or of order the vev of some $F_{i}$ (when one of their $U(1)_{F_{i}}$ charges differs by one). We assume that both the $F_{i}$ vevs and $M$ are of order a TeV. Any residual anomaly in the $U(1)_{F_{i}}$ symmetries can be handled either by introducing more heavy fermions or using the Green-Schwarz mechanism at the TeV scale.

We do not suggest that this model is the most efficient one implementing the basic concepts of our proposal. We have made an explicit trade-off, in some sense, of maximizing the number of the new heavy fermions required in order to minimize the complexity of the messenger sector and the charge assignments.

With the charge assignments listed in Table 3 the only dimension 4 couplings of fermions to the Higgs scalar are

$$
\begin{aligned}
& f_{1} \bar{q}_{3 L} u_{3 R} \bar{H}+f_{2} \bar{Q}_{2 L} U_{2 R} \bar{H}+f_{3} \bar{Q}_{4 R} U_{4 L} \bar{H}+f_{4} \bar{Q}_{6 L} U_{3 R} \bar{H} \\
& +f_{5} \bar{Q}_{7 L} U_{6 R} \bar{H}+f_{6} \bar{Q}_{3 L} D_{3 R} H+f_{7} \bar{Q}_{4 L} D_{4 R} H+f_{8} \bar{Q}_{7 L} D_{7 R} H+\text { h.c. }
\end{aligned}
$$

The only dimension 4 couplings of fermions to the the $S$ messenger scalar are

$$
\begin{aligned}
& f_{9} \bar{q}_{1 L} Q_{1 R} S^{\dagger}+f_{10} \bar{q}_{2 L} Q_{2 R} S^{\dagger}+f_{11} \bar{q}_{3 L} Q_{3 R} S^{\dagger}+f_{12} \bar{U}_{1 L} u_{1 R} S \\
& +f_{13} \bar{U}_{2 L} u_{2 R} S+f_{14} \bar{D}_{1 L} d_{1 R} S+f_{15} \bar{D}_{2 L} d_{2 R} S+f_{16} \bar{D}_{3 L} d_{3 R} S \\
& +f_{17} \bar{Q}_{2 L} Q_{4 R} S^{\dagger}+f_{18} \bar{Q}_{1 L} Q_{5 R} S^{\dagger}+f_{19} \bar{Q}_{7 L} Q_{5 R} S^{\dagger}+f_{20} \bar{Q}_{5 L} Q_{7 R} S^{\dagger} \\
& +f_{21} \bar{U}_{4 L} U_{2 R} S+f_{22} \bar{U}_{5 L} U_{1 R} S+f_{23} \bar{U}_{6 L} U_{5 R} S+f_{24} \bar{D}_{4 L} D_{3 R} S \\
& +f_{25} \bar{D}_{3 L} D_{4 R} S^{\dagger}+f_{26} \bar{D}_{5 L} D_{2 R} S+f_{27} \bar{D}_{6 L} D_{1 R} S+f_{28} \bar{D}_{1 L} D_{6 R} S^{\dagger} \\
& +f_{29} \bar{D}_{7 L} D_{5 R} S+f_{30} \bar{D}_{8 L} D_{6 R} S+f_{31} \bar{D}_{6 L} D_{8 R} S^{\dagger}+\text { h.c. }
\end{aligned}
$$

The direct fermion mass terms and mixings consistent with the flavon symmetries and SM gauge symmetries generated by operators of dimension 4 or less are

$$
\begin{aligned}
& f_{32} \bar{Q}_{1 L} Q_{1 R} F_{3}^{\dagger}+f_{33} \bar{Q}_{2 L} Q_{2 R} F_{1}+f_{34} \bar{Q}_{3 L} Q_{3 R} F_{1}+f_{35} \bar{Q}_{3 L} Q_{3 R} F_{2}^{\dagger} \\
& +f_{36} \bar{Q}_{4 L} Q_{4 R} F_{2}^{\dagger}+f_{37} \bar{Q}_{5 L} Q_{5 R} F_{1}+f_{38} \bar{Q}_{5 L} Q_{6 R} F_{2}+f_{39} \bar{Q}_{6 L} Q_{6 R} F_{3}^{\dagger} \\
& +f_{40} \bar{Q}_{7 L} Q_{7 R} F_{1}^{\dagger}+f_{41} \bar{U}_{1 L} U_{1 R} F_{3}+f_{42} \bar{U}_{2 L} U_{2 R} F_{2}^{\dagger}+M \bar{U}_{3 L} U_{3 R}+f_{43} \bar{U}_{3 L} U_{4 R} F_{3}^{\dagger} \\
& +f_{44} \bar{U}_{4 L} U_{4 R} F_{1}^{\dagger}+f_{45} \bar{U}_{5 L} U_{5 R} F_{2}^{\dagger}+f_{46} \bar{U}_{6 L} U_{6 R} F_{1}^{\dagger}+f_{47} \bar{D}_{1 L} D_{1 R} F_{3}^{\dagger} \\
& +f_{48} \bar{D}_{2 L} D_{2 R} F_{1}^{\dagger}+M \bar{D}_{3 L} D_{3 R}+M \bar{D}_{4 L} D_{4 R}+f_{49} \bar{D}_{5 L} D_{5 R} F_{3} \\
& +f_{50} \bar{D}_{4 L} D_{5 R} F_{2}^{\dagger}+f_{51} \bar{D}_{6 L} D_{6 R} F_{3}+f_{52} \bar{D}_{7 L} D_{7 R} F_{2}^{\dagger}+M \bar{D}_{8 L} D_{7 R}+f_{53} \bar{D}_{8 L} D_{8 R} F_{3}^{\dagger}+\text { h.c., }
\end{aligned}
$$

where for simplicity of notation we have used $M$ to denote all the $\mathrm{TeV}$ scale mass parameters.

Thus, integrating out the heavy fermions in the tree level diagram composed from the couplings

$$
f_{11} \bar{q}_{3 L} Q_{3 R} S^{\dagger}+f_{34}^{*} \bar{Q}_{3 R} Q_{3 L} F_{1}^{\dagger}+f_{10} \bar{Q}_{3 L} D_{3 R} H+M \bar{D}_{3 R} D_{3 L}+f_{3} \bar{D}_{3 L} d_{3 R} S
$$

produces an effective coupling below the $\mathrm{TeV}$ scale proportional to

$$
f_{11} f_{34}^{*} f_{10} f_{3} \frac{F_{1}^{\dagger}}{M} \frac{S^{\dagger} S}{M^{2}} \bar{q}_{3 L} d_{3 R} H+\text { h.c. . }
$$




\begin{tabular}{|c|c|c|c|c|c||c|c|c|c|c|c|}
\hline Field & $\mathbf{U}(\mathbf{1})_{\mathbf{Y}}$ & $\mathbf{U}(\mathbf{1})_{\mathbf{S}}$ & $\mathbf{U}(\mathbf{1})_{\mathbf{F} 1}$ & $\mathbf{U}(\mathbf{1})_{\mathbf{F} 2}$ & $\mathbf{U}(\mathbf{1})_{\mathbf{F} 3}$ & $\mathbf{F i e l d}$ & $\mathbf{U}(\mathbf{1})_{\mathbf{Y}}$ & $\mathbf{U}(\mathbf{1})_{\mathbf{S}}$ & $\mathbf{U}(\mathbf{1})_{\mathbf{F} \mathbf{1}}$ & $\mathbf{U}(\mathbf{1})_{\mathbf{F} 2}$ & $\mathbf{U}(\mathbf{1})_{\mathbf{F} 3}$ \\
\hline$q_{1 L}$ & $1 / 6$ & 0 & 1 & 2 & 1 & $U_{1 L}$ & $2 / 3$ & 1 & 0 & 1 & 1 \\
$q_{2 L}$ & $1 / 6$ & 0 & 0 & 1 & 0 & $U_{1 R}$ & $2 / 3$ & 1 & 0 & 1 & 0 \\
$q_{3 L}$ & $1 / 6$ & 0 & 0 & 0 & 0 & $U_{2 L}$ & $2 / 3$ & 1 & 1 & 0 & 0 \\
$u_{1 R}$ & $2 / 3$ & 0 & 0 & 1 & 1 & $U_{2 R}$ & $2 / 3$ & 1 & 1 & 1 & 0 \\
$u_{2 R}$ & $2 / 3$ & 0 & 1 & 0 & 0 & $U_{3 L}$ & $2 / 3$ & 2 & 2 & 1 & -1 \\
$u_{3 R}$ & $2 / 3$ & 0 & 0 & 0 & 0 & $U_{3 R}$ & $2 / 3$ & 2 & 2 & 1 & -1 \\
$d_{1 R}$ & $-1 / 3$ & 0 & 1 & 2 & 0 & $U_{4 L}$ & $2 / 3$ & 2 & 1 & 1 & 0 \\
$d_{2 R}$ & $-1 / 3$ & 0 & 0 & 1 & 1 & $U_{4 R}$ & $2 / 3$ & 2 & 2 & 1 & 0 \\
$d_{3 R}$ & $-1 / 3$ & 0 & 1 & 0 & 0 & $U_{5 L}$ & $2 / 3$ & 2 & 0 & 1 & 0 \\
$Q_{1 L}$ & $1 / 6$ & 1 & 1 & 2 & 0 & $U_{5 R}$ & $2 / 3$ & 2 & 0 & 2 & 0 \\
$Q_{1 R}$ & $1 / 6$ & 1 & 1 & 2 & 1 & $U_{6 L}$ & $2 / 3$ & 3 & 0 & 2 & 0 \\
$Q_{2 L}$ & $1 / 6$ & 1 & 1 & 1 & 0 & $U_{6 R}$ & $2 / 3$ & 3 & 1 & 2 & 0 \\
$Q_{2 R}$ & $1 / 6$ & 1 & 0 & 1 & 0 & $D_{1 L}$ & $-1 / 3$ & 1 & 1 & 2 & 0 \\
$Q_{3 L}$ & $1 / 6$ & 1 & 1 & 0 & 0 & $D_{1 R}$ & $-1 / 3$ & 1 & 1 & 2 & 1 \\
$Q_{3 R}$ & $1 / 6$ & 1 & 0 & 0 & 0 & $D_{2 L}$ & $-1 / 3$ & 1 & 0 & 1 & 1 \\
$Q_{4 L}$ & $1 / 6$ & 2 & 1 & 0 & 0 & $D_{2 R}$ & $-1 / 3$ & 1 & 1 & 1 & 1 \\
$Q_{4 R}$ & $1 / 6$ & 2 & 1 & 1 & 0 & $D_{3 L}$ & $-1 / 3$ & 1 & 1 & 0 & 0 \\
$Q_{5 L}$ & $1 / 6$ & 2 & 2 & 2 & 0 & $D_{3 R}$ & $-1 / 3$ & 1 & 1 & 0 & 0 \\
$Q_{5 R}$ & $1 / 6$ & 2 & 1 & 2 & 0 & $D_{4 L}$ & $-1 / 3$ & 2 & 1 & 0 & 0 \\
$Q_{6 L}$ & $1 / 6$ & 2 & 2 & 1 & -1 & $D_{4 R}$ & $-1 / 3$ & 2 & 1 & 0 & 0 \\
$Q_{6 R}$ & $1 / 6$ & 2 & 2 & 1 & 0 & $D_{5 L}$ & $-1 / 3$ & 2 & 1 & 1 & 1 \\
$Q_{7 L}$ & $1 / 6$ & 3 & 1 & 2 & 0 & $D_{5 R}$ & $-1 / 3$ & 2 & 1 & 1 & 0 \\
$Q_{7 R}$ & $1 / 6$ & 3 & 2 & 2 & 0 & $D_{6 L}$ & $-1 / 3$ & 2 & 1 & 2 & 1 \\
$H$ & $1 / 2$ & 0 & 0 & 0 & 0 & $D_{6 R}$ & $-1 / 3$ & 2 & 1 & 2 & 0 \\
$S$ & 0 & 1 & 0 & 0 & 0 & $D_{7 L}$ & $-1 / 3$ & 3 & 1 & 1 & 0 \\
$F_{1}$ & 0 & 0 & 1 & 0 & 0 & $D_{7 R}$ & $-1 / 3$ & 3 & 1 & 2 & 0 \\
$F_{2}$ & 0 & 0 & 0 & 1 & 0 & $D_{8 L}$ & $-1 / 3$ & 3 & 1 & 2 & 0 \\
$F_{3}$ & 0 & 0 & 0 & 0 & 1 & $D_{8 R}$ & $-1 / 3$ & 3 & 1 & 2 & 1 \\
\hline
\end{tabular}

Table 3: Charge assignments in the three generation model for the scalar fields $H, S, F_{i}$, the SM quark fields $q_{i L}, u_{i R}, d_{i R}$, and the heavy quark pairs $Q_{i L}, Q_{i R}, U_{i L}, U_{i R}, D_{i L}, D_{i R}$. 
Integrating out the heavy fermions in the tree level diagram composed from the couplings

$$
f_{10} \bar{q}_{2 L} Q_{2 R} S^{\dagger}+f_{33}^{*} \bar{Q}_{2 R} Q_{2 L} F_{1}^{\dagger}+f_{2} \bar{Q}_{2 L} U_{2 R} \bar{H}+f_{42}^{*} \bar{U}_{2 R} U_{2 L} F_{2}+f_{13} \bar{U}_{2 L} u_{2 R} S
$$

produces an effective coupling below the $\mathrm{TeV}$ scale proportional to

$$
f_{10} f_{33}^{*} f_{2} f_{42}^{*} f_{13} \frac{F_{1}^{\dagger} F_{2}}{M^{2}} \frac{S^{\dagger} S}{M^{2}} \bar{q}_{2 L} u_{2 R} \bar{H}+\text { h.c. . }
$$

Integrating out the heavy fermions in the tree level diagram composed from the couplings

$$
\begin{aligned}
& f_{10} \bar{q}_{2 L} Q_{2 R} S^{\dagger}+f_{33}^{*} \bar{Q}_{2 R} Q_{2 L} F_{1}^{\dagger}+f_{17} \bar{Q}_{2 L} Q_{4 R} S^{\dagger}+f_{36}^{*} \bar{Q}_{4 R} Q_{4 L} F 2+f_{7} \bar{Q}_{4 L} D_{4 R} H \\
& +M \bar{D}_{4 R} D_{4 L}+f_{24}^{*} \bar{D}_{4 L} D_{3 R} S+M \bar{D}_{3 R} D_{3 L}+f_{3} \bar{D}_{3 L} d_{3 R} S
\end{aligned}
$$

produces an effective coupling below the $\mathrm{TeV}$ scale proportional to

$$
f_{10} f_{33}^{*} f_{17} f_{36}^{*} f_{7} f_{24}^{*} f_{3} \frac{F_{1}^{\dagger} F_{2}}{M^{2}} \frac{\left(S^{\dagger} S\right)^{2}}{M^{4}} \bar{q}_{2 L} d_{3 R} H+\text { h.c. . }
$$

Integrating out the heavy fermions in the tree level diagram composed from the couplings

$$
\begin{aligned}
& f_{11} \bar{q}_{3 L} Q_{3 R} S^{\dagger}+f_{34}^{*} \bar{Q}_{3 R} Q_{3 L} F_{1}^{\dagger}+f_{10} \bar{Q}_{3 L} D_{3 R} H+f_{24}^{*} \bar{D}_{3 R} D_{4 L} S^{\dagger}+f_{50} \bar{D}_{4 L} D_{5 R} F_{2}^{\dagger} \\
& +f_{49}^{*} \bar{D}_{5 R} D_{5 L} F_{3}^{\dagger}+f_{26} \bar{D}_{5 L} D_{2 R} S+f_{48}^{*} \bar{D}_{2 R} D_{2 L} F_{1}+f_{15} \bar{D}_{2 L} d_{2 R} S
\end{aligned}
$$

produces an effective coupling below the $\mathrm{TeV}$ scale proportional to

$$
f_{11} f_{34}^{*} f_{10} f_{24}^{*} f_{50} f_{49}^{*} f_{26} f_{48}^{*} f_{15} \frac{F_{1}^{\dagger} F_{1} F_{2}^{\dagger} F_{3}^{\dagger}}{M^{4}} \frac{\left(S^{\dagger} S\right)^{2}}{M^{4}} \bar{q}_{3 L} d_{2 R} H+h . c . .
$$

There is also another tree level contribution to $h_{32}^{d}$, proportional to

$$
f_{11} f_{34}^{*} f_{10} f_{24} f_{50} f_{49}^{*} f_{26} f_{48}^{*} f_{15} \frac{F_{1}^{\dagger} F_{1} F_{2}^{\dagger} F_{3}^{\dagger}}{M^{4}} \frac{\left(S^{\dagger} S\right)^{2}}{M^{4}} \bar{q}_{3 L} d_{2 R} H+\text { h.c. . }
$$

Integrating out the heavy fermions in the tree level diagram composed from the couplings

$$
\begin{aligned}
& f_{10} \bar{q}_{2 L} Q_{2 R} S^{\dagger}+f_{33}^{*} \bar{Q}_{2 R} Q_{2 L} F_{1}^{\dagger}+f_{17} \bar{Q}_{2 L} Q_{4 R} S^{\dagger}+f_{36}^{*} \bar{Q}_{4 R} Q_{4 L} F 2+f_{7} \bar{Q}_{4 L} D_{4 R} H+M \bar{D}_{4 R} D_{4 L} \\
& +f_{50} \bar{D}_{4 L} D_{5 R} F_{2}^{\dagger}+f_{49}^{*} \bar{D}_{5 R} D_{5 L} F_{3}^{\dagger}+f_{26} \bar{D}_{5 L} D_{2 R} S+f_{48}^{*} \bar{D}_{2 R} D_{2 L} F_{1}+f_{15} \bar{D}_{2 L} d_{2 R} S
\end{aligned}
$$

produces an effective coupling below the $\mathrm{TeV}$ scale proportional to

$$
f_{10} f_{33}^{*} f_{17} f_{36}^{*} f_{7} f_{50} f_{49}^{*} f_{26} f_{48}^{*} f_{15} \frac{F_{1}^{\dagger} F_{1} F_{2}^{\dagger} F_{2} F_{3}^{\dagger}}{M^{5}} \frac{\left(S^{\dagger} S\right)^{2}}{M^{4}} \bar{q}_{3 L} d_{2 R} H+\text { h.c. . }
$$

Integrating out the heavy fermions in the tree level diagram composed from the couplings

$$
\begin{aligned}
& f_{9} \bar{q}_{1 L} Q_{1 R} S^{\dagger}+f_{32}^{*} \bar{Q}_{1 R} Q_{1 L} F_{3}+f_{18}^{*} \bar{Q}_{1 L} Q_{5 R} S^{\dagger}+f_{19}^{*} \bar{Q}_{5 R} Q_{7 L} S^{\dagger}+f_{8} \bar{Q}_{7 L} D_{7 R} H \\
& +M \bar{D}_{7 R} D_{8 L}+f_{30} \bar{D}_{8 L} D_{6 R} S+f_{28} \bar{D}_{6 R} D_{1 L} S+f_{14} \bar{D}_{1 L} d_{1 R} S
\end{aligned}
$$


produces an effective coupling below the $\mathrm{TeV}$ scale proportional to

$$
f_{9} f_{32}^{*} f_{18}^{*} f_{19}^{*} f_{8} f_{30} f_{28} f_{14} \frac{F_{3}}{M} \frac{\left(S^{\dagger} S\right)^{3}}{M^{6}} \bar{q}_{1 L} d_{1 R} H+\text { h.c. . }
$$

There are four other very similar tree level contributions to $h_{11}^{d}$.

Integrating out the heavy fermions in the tree level diagram composed from the couplings

$$
\begin{aligned}
& f_{9} \bar{q}_{1 L} Q_{1 R} S^{\dagger}+f_{32}^{*} \bar{Q}_{1 R} Q_{1 L} F_{3}+f_{18}^{*} \bar{Q}_{1 L} Q_{5 R} S^{\dagger}+f_{19}^{*} \bar{Q}_{5 R} Q_{7 L} S^{\dagger}+f_{5} \bar{Q}_{7 L} U_{6 R} \bar{H}+f_{46}^{*} \bar{U}_{6 R} U_{6 L} F_{1} \\
& +f_{23} \bar{U}_{6 L} U_{5 R} S+f_{45}^{*} \bar{U}_{5 R} U_{5 L} F_{2}+f_{22} \bar{U}_{5 L} U_{1 R} S+f_{41}^{*} \bar{U}_{1 R} U_{1 L} F_{3}^{\dagger}+f_{12} \bar{U}_{1 L} u_{1 R} S
\end{aligned}
$$

produces an effective coupling below the $\mathrm{TeV}$ scale proportional to

$$
f_{9} f_{32}^{*} f_{18}^{*} f_{19}^{*} f_{5} f_{46}^{*} f_{23} f_{45}^{*} f_{22} f_{41}^{*} f_{12} \frac{F_{1} F_{2} F_{3}^{\dagger} F_{3}}{M^{4}} \frac{\left(S^{\dagger} S\right)^{3}}{M^{6}} \bar{q}_{1 L} u_{1 R} \bar{H}+\text { h.c. . }
$$

Integrating out the heavy fermions in the tree level diagram composed from the couplings

$$
\begin{aligned}
& f_{9} \bar{q}_{1 L} Q_{1 R} S^{\dagger}+f_{32}^{*} \bar{Q}_{1 R} Q_{1 L} F_{3}+f_{18}^{*} \bar{Q}_{1 L} Q_{5 R} S^{\dagger}+f_{19}^{*} \bar{Q}_{5 R} Q_{7 L} S^{\dagger}+f_{8} \bar{Q}_{7 L} D_{7 R} H+f_{52}^{*} \bar{D}_{7 R} D_{7 L} F_{2} \\
& +f_{29} \bar{D}_{7 L} D_{5 R} S+f_{49}^{*} \bar{D}_{5 R} D_{5 L} F_{3}^{\dagger}+f_{26} \bar{D}_{5 L} D_{2 R} S+f_{48}^{*} \bar{D}_{2 R} D_{2 L} F_{1}+f_{15} \bar{D}_{2 L} d_{2 R} S
\end{aligned}
$$

produces an effective coupling below the $\mathrm{TeV}$ scale proportional to

$$
f_{9} f_{32}^{*} f_{18}^{*} f_{19}^{*} f_{8} f_{52}^{*} f_{29} f_{49}^{*} f_{26} f_{48}^{*} f_{15} \frac{F_{1} F_{2} F_{3}^{\dagger} F_{3}}{M^{4}} \frac{\left(S^{\dagger} S\right)^{3}}{M^{6}} \bar{q}_{1 L} d_{2 R} H+\text { h.c. . }
$$

Integrating out the heavy fermions in the tree level diagram composed from the couplings

$$
\begin{aligned}
& f_{9} \bar{q}_{1 L} Q_{1 R} S^{\dagger}+f_{32}^{*} \bar{Q}_{1 R} Q_{1 L} F_{3}+f_{18}^{*} \bar{Q}_{1 L} Q_{5 R} S^{\dagger}+f_{19}^{*} \bar{Q}_{5 R} Q_{7 L} S^{\dagger}+f_{8} \bar{Q}_{7 L} D_{7 R} H+f_{52}^{*} \bar{D}_{7 R} D_{7 L} F_{2} \\
& +f_{29} \bar{D}_{7 L} D_{5 R} S+f_{50}^{*} \bar{D}_{5 R} D_{4 L} F_{2}+f_{24}^{*} \bar{D}_{4 L} D_{3 R} S+M \bar{D}_{3 R} D_{3 L}+f_{3} \bar{D}_{3 L} d_{3 R} S
\end{aligned}
$$

produces an effective coupling below the $\mathrm{TeV}$ scale proportional to

$$
f_{9} f_{32}^{*} f_{18}^{*} f_{19}^{*} f_{8} f_{52}^{*} f_{29} f_{50}^{*} f_{24}^{*} f_{3} \frac{\left(F_{2}\right)^{2} F_{3}}{M^{3}} \frac{\left(S^{\dagger} S\right)^{3}}{M^{6}} \bar{q}_{1 L} d_{3 R} H+\text { h.c. . }
$$

There is one other very similar tree level contribution to $h_{13}^{d}$.

Integrating out the heavy fermions in the tree level diagram composed from the couplings

$$
\begin{aligned}
& f_{11} \bar{q}_{3 L} Q_{3 R} S^{\dagger}+f_{34}^{*} \bar{Q}_{3 R} Q_{3 L} F_{1}^{\dagger}+f_{10} \bar{Q}_{3 L} D_{3 R} H+f_{24}^{*} \bar{D}_{3 R} D_{4 L} S^{\dagger}+f_{50} \bar{D}_{4 L} D_{5 R} F_{2}^{\dagger}+f_{29}^{*} \bar{D}_{5 R} D_{7 L} S^{\dagger} \\
& +f_{52}^{*} \bar{D}_{7 L} D_{7 R} F_{2}^{\dagger}+M \bar{D}_{7 R} D_{8 L}+f_{30} \bar{D}_{8 L} D_{6 R} S+f_{28} \bar{D}_{6 R} D_{1 L} S+f_{14} \bar{D}_{1 L} d_{1 R} S
\end{aligned}
$$

produces an effective coupling below the $\mathrm{TeV}$ scale proportional to

$$
f_{11} f_{34}^{*} f_{10} f_{24}^{*} f_{50} f_{29}^{*} f_{52}^{*} f_{30} f_{28} f_{14} \frac{F_{1}^{\dagger}\left(F_{2}^{\dagger}\right)^{2}}{M^{3}} \frac{\left(S^{\dagger} S\right)^{3}}{M^{6}} \bar{q}_{3 L} d_{1 R} H+\text { h.c. . }
$$

The following effective couplings are not generated or are generated at higher order in $\epsilon$ and/or $\beta$ : $h_{23}^{u}$, $h_{32}^{u}, h_{12}^{u}, h_{21}^{u}, h_{13}^{u}, h_{31}^{u}$, and $h_{21}^{d}$. As already indicated these couplings are not needed to reproduce the observed SM quark masses and mixings. For illustration, $h_{32}^{u}$ arises from the effective coupling

$$
f_{11} f_{34}^{*} f_{6} f_{25} f_{7}^{*} f_{36} f_{17}^{*} f_{2} f_{42}^{*} f_{13} \frac{F_{1}^{\dagger} F_{2} F_{2}^{\dagger}}{M^{3}} \frac{\left(S^{\dagger} S\right)^{2}}{M^{4}} \frac{H^{\dagger} H}{M^{2}} \bar{q}_{3 L} u_{2 R} \bar{H}+\text { h.c. }
$$


so the extra suppression relative to eq. (1) is by an additional factor of $\beta$ as well as an additional factor of $\epsilon$.

Since $h_{12}^{u}$ and $h_{21}^{u}$ have extra suppression in this model, $D^{0}-\overline{D^{0}}$ mixing also has extra suppression. This weakens the lower bound on $m_{s}$ derived in Section 3.2 . Similarly since $h_{23}^{u}$ and $h_{32}^{u}$ have extra suppression the relatively large BR for $t \rightarrow c s$ discussed in Section 4.2 will not occur for this particular realization.

\section{Conclusion}

We have presented a framework in which only the top quark obtains its mass from the Yukawa interaction with the SM Higgs boson via dimension four operators. All the other quarks receive their masses from operators of dimension six or higher involving a complex scalar $S$ that is part of an extended Higgs sector and whose vev is at the electroweak scale. The successive hierarchy of light quark masses is generated via the expansion parameter $\left(\frac{S^{\dagger} S}{M^{2}}\right) \sim \epsilon^{2}$, where $\epsilon \equiv \frac{v_{s}}{M} \sim 0.15$. All the couplings of the higher dimensional operators are of order one. We are able to generate the appropriate hierarchy of fermion masses with this small parameter $\epsilon$. Since $v_{s}$ is at the EW scale, the physics of the new scale $M$ is not far above a TeV. We predict a neutral scalar $s$, which gives rise to signals that could be detected at the LHC or at the Tevatron. We make new predictions for Higgs decays and for top decays. The model has a light $Z^{\prime}$ that has very weak couplings to SM fermions, but could be light enough to be produced via mixing in Higgs decays at the LHC; this could give rise to invisible Higgs decays, displaced vertices from the $Z^{\prime}$ decays, or multilepton final states, depending on the mass and lifetime of the $Z^{\prime}$.

We have presented an explicit model in which the effective interaction given in (11) is realized. This involves extending the SM gauge symmetry by an abelian gauge symmetry $U(1)_{S}$ and a local flavon symmetry group $U(1)_{F_{1}} \times U(1)_{F_{2}} \times U(1)_{F_{3}}$ The flavon symmetry is spontaneously broken at the TeV scale by a complex flavon scalars $F_{1}, F_{2}, F_{3}$, whereas the $U(1)_{S}$ symmetry is broken at the electroweak scale by the complex scalar $S$, which is a SM singlet extension of the SM Higgs sector. $S$ acts as the messenger of both flavor and electroweak symmetry breaking. The model requires the existence of vectorlike quarks and leptons, both EW doublets and singlets, at the TeV scale. These can be probed at the LHC. Their decays will be a new source for Higgs production and give rise to final states with four $Z$ 's or four $Z$ 's and other interesting new physics signals at the LHC.

We have restricted ourselves to models where all of the hierarchies of the SM quark and charged lepton masses and mixings arise from powers of the vev of a single messenger field. In [6], a framework was suggested in which all of these hierarchies arise from powers of $\beta=\left(\frac{H^{\dagger} H}{M^{2}}\right)$. As we saw in the previous section, in explicit models it is natural to generate powers of both $\epsilon$ and $\beta$. Thus the model presented here and the framework of [6] are two extremes of a more general class of models. Obviously one could also generalize by introducing a more complicated messenger sector, i.e. further extending the Higgs sector.

A truely viable model should have fewer species of heavy fermions than were required in our example, ameliorating what is otherwise a dramatic worsening of the little hierarchy problem of the Standard Model. This could be achieved by a more efficient construction of the messenger sector and its interplay with the flavon sector. Another interesting direction is to attempt to generate some of the higher order effective couplings from the top quark Yukawa, as was done successfully with leptoquark-generated loop diagrams in [4. 


\section{Acknowledgements}

We are grateful to Bogdan Dobrescu for several useful discussions. SN and ZM would like to thank the Fermilab Theoretical Physics Department for warm hospitality and support during the completion of this work. This research was supported in part by grant numbers DOE-FG02-04ER41306 and DOE-FG02ER46140. Fermilab is operated by the Fermi Research Alliance LLC under contract DE-AC02-07CH11359 with the U.S. Dept. of Energy.

\section{References}

[1] C. D. Froggatt and H. B. Nielsen, Nucl. Phys. B 147, 277 (1979).

[2] There is a vast literature on the fermion mass hierarchy problem; see for example:

A. De Rujula, H. Georgi and S. L. Glashow, Annals Phys. 109, 258 (1977);

H. Georgi and C. Jarlskog, Phys. Lett. B 86, 297 (1979);

S.M. Barr, Phys. Rev. D21, 1424 (1980);

C. T. Hill, Phys. Rev. D 24, 691 (1981);

H. Georgi, A. Manohar and A. Nelson, Phys. Lett. 126B, 169 (1983);

S. Dimopoulos, Phys. Lett. B 129, 417 (1983);

J. Bagger, S. Dimopoulos, E. Masso and M. H. Reno, Nucl. Phys. B 258, 565 (1985);

K.S. Babu and R.N. Mohapatra, Phys. Rev. Lett. 64, 2747 (1990);

M. Leurer, Y. Nir and N. Seiberg, Nucl. Phys. B 398, 319 (1993);

Z. Berezhiani and R. Rattazzi, Nucl.Phys. B407, 249 (1993);

Y. Nir and N. Seiberg, Phys. Lett. B309, 337 (1993);

L. E. Ibanez and G. G. Ross, Phys. Lett. B 332, 100 (1994);

P. Binetruy and P. Ramond, Phys. Lett. B 350, 49 (1995);

E. Dudas, S. Pokorski and C. A. Savoy, Phys. Lett. B 356, 45 (1995);

C. H. Albright and S. Nandi, Mod. Phys. Lett. A 11, 737 (1996);

D. J. Muller and S. Nandi, Phys. Lett. B 383, 345 (1996).

[3] S. Nandi and Z. Tavartkiladze, arXiv:0804.1996 [hep-ph].

[4] B. A. Dobrescu and P. J. Fox, JHEP 0808, 100 (2008) arXiv:0805.0822 [hep-ph]].

[5] B. S. Balakrishna, Phys. Rev. Lett. 60, 1602 (1988); B. S. Balakrishna, A. L. Kagan and R. N. Mohapatra, Phys. Lett. B 205, 345 (1988); B. S. Balakrishna and R. N. Mohapatra, Phys. Lett. B 216, 349 (1989).

[6] K. S. Babu and S. Nandi, Phys. Rev. D 62, 033002 (2000) arXiv:hep-ph/9907213.

[7] G. F. Giudice and O. Lebedev, Phys. Lett. B 665, 79 (2008) arXiv:0804.1753 [hep-ph]].

[8] I. Dorsner and S. M. Barr, Phys. Rev. D 65, 095004 (2002) arXiv:hep-ph/0201207.

[9] W.-M.Yao et al. (Particle Data Group), J. Phys. G 33, 1 (2006) and 2007 partial update for the 2008 edition.

[10] T. Aaltonen et al. [CDF Collaboration], Phys. Rev. Lett. 100, 121802 (2008) [arXiv:0712.1567 [hep-ex]]. K. Abe et al. [BELLE Collaboration], Phys. Rev. Lett. 99, 131803 (2007) arXiv:0704.1000 [hep-ex]]. 
[11] LEP Electroweak Working Group, http://lepewwg.web.cern.ch.

[12] P. Langacker, arXiv:0801.1345 [hep-ph].

[13] B. A. Dobrescu, Phys. Rev. Lett. 94, 151802 (2005) arXiv:hep-ph/0411004.

[14] S. Mrenna and J. D. Wells, Phys. Rev. D 63, 015006 (2001) arXiv:hep-ph/0001226.

A. Melnitchouk [D0 Collaboration], Int. J. Mod. Phys. A 20, 3305 (2005) arXiv:hep-ex/0501067.

[15] G. Bernardi et al. [Tevatron New Phenomena Higgs Working Group and CDF Collaboration and D], arXiv:0808.0534 [hep-ex].

[16] J. A. Aguilar-Saavedra and G. C. Branco, Phys. Lett. B 495, 347 (2000) arXiv:hep-ph/0004190.

[17] M. Cacciari, S. Frixione, M. M. Mangano, P. Nason and G. Ridolfi, arXiv:0804.2800 [hep-ph].

[18] All plots of Higgs branching ratios were made by modifying the HDecay code:

A.Djouadi, J.Kalinowski and M.Spira, Comp. Phys. Commun. 108 C (1998) 56, hep-ph/9704448. 